\title{
Liaisons dangereuses: Increasing connectivity, risk sharing, and systemic risk
}

\author{
Stefano Battiston ${ }^{\mathrm{a}, *}$, Domenico Delli Gatti ${ }^{\mathrm{b}}$, Mauro Gallegati ${ }^{\mathrm{c}}$, \\ Bruce Greenwald ${ }^{\mathrm{d}}$, Joseph E. Stiglitz ${ }^{\mathrm{d}}$ \\ ${ }^{\text {a }}$ ETH Zurich, Switzerland \\ ${ }^{\mathrm{b}}$ Catholic University of Milan, Italy \\ c Polytechnic University of Marche, Ancona, Italy \\ ${ }^{\mathrm{d}}$ Columbia University, NY, USA
}

\section{A R T I C L E I N F O}

Available online 12 April 2012

JEL classification:

D85

G01

G21

Keywords:

Systemic risk

Network models

Contagion

Financial acceleration

Financial crisis

\begin{abstract}
A B S T R A C T
The recent financial crisis poses the challenge to understand how systemic risk arises endogenously and what architecture can make the financial system more resilient to global crises. This paper shows that a financial network can be most resilient for intermediate levels of risk diversification, and not when this is maximal, as generally thought so far. This finding holds in the presence of the financial accelerator, i.e. when negative variations in the financial robustness of an agent tend to persist in time because they have adverse effects on the agent's subsequent performance through the reaction of the agent's counterparties.
\end{abstract}

(c) 2012 Elsevier B.V. All rights reserved.

\section{Introduction}

The recent financial crisis has shown that systemic risk has been dramatically underestimated (Bartram et al., 2007). Understanding the nature of systemic risk is crucial if appropriate policy responses are to be framed, especially since the impact of financial shocks on the real economy increases with the depth of the financial system (Kroszner et al., 2007). Moreover, it has been recognized that financial crises are characterized by the procyclicality of balance sheets and leverage across financial institutions. This is the case especially when they get connected to each other in the process of originating and distributing securities (Brunnermeier, 2008; Morris and Shin, 2008; Shleifer and Vishny, 2010). In general, financial ties of various types among institutions have in principle an ambiguous effect, since they allow for the diversification of risk, but open also the door to financial contagion (Allen and Gale, 2001). There is strong empirical evidence that in the unraveling of the recent crisis, contagion played a large role, propagated primarily through liquidity and risk-premium channels (Longstaff, 2010). The recent financial crisis has brought to the fore the crucial question of the relationship between the architecture of the global financial system and systemic risk (Allen et al., 2011). The common view in the literature has been so far that the beneficial effect of diversification always prevails and more integrated systems are more resilient (Allen and Gale, 2001). In contrast, a very recent stream of work has found conditions on the propagation of financial contagion and bankruptcies so that full risk diversification or full financial integration is not optimal (Allen et al., 2011; Castiglionesi and Navarro, 2010; Wagner, 2010; Stiglitz, 2010, forthcoming).

\footnotetext{
* Corresponding author.

E-mail address: sbattiston@ethz.ch (S. Battiston).
} 
This paper contributes to the debate on the resilience of financial networks by introducing a dynamic model for the evolution of financial robustness. We show that, in the presence of financial acceleration and persistence - i.e. when the variations in the level of financial robustness of institutions tend to persist in time or get amplified - the probability of default does not decrease monotonically with diversification. As a result, the financial network is most resilient for an intermediate level of connectivity.

Our work is related to several streams of the literature. We follow the standard approach in finance of modeling the default of one firm as a problem of first passage time in a stochastic diffusion process representing the valuation of corporate liabilities (Merton, 1974; Black and Cox, 1976; Longstaff and Schwartz, 1995). In particular, the index representing the distance to default or the level of creditworthiness (Hull and White, 2000) is assumed to evolve over time as a stochastic differential equation (SDE) with a lower absorbing barrier, so that the firm defaults when the index value becomes zero (or hits from above a positive "bankruptcy" threshold). In addition, one can compute numerically the probability of joint default of several firms in the presence of correlations among their level of creditworthiness (Hull and White, 2001; Zhou, 1997). However, key to the analysis of systemic risk is the endogenous determination of correlations arising from the external effects that the distress of an agent causes to her counterparties.

Several types of external effects have been investigated in the literature. On the one hand, the failure of one bank adversely affects other banks-financial contagion (Diamond and Dybvig, 1983; Allen and Gale, 2001; Stiglitz and Greenwald, 2003). This, in turn, can occur through several channels: (a) bank runs-where the failure of one bank induces depositors of other banks to withdraw their funds; (b) interbank lending-where the failure of one bank implies that others will not be fully repaid; and (c) real economic activity-where the failure of one bank has adverse effects on other bank borrowers, lowering their probability of repayment. On the other hand, agents may be adversely affected by the depreciation of a common asset-asset price contagion (Kiyotaki and Moore, 2002, 1997). Again, this can occur through several channels, the most important of which may be through the value of collateral, the impact of reduced collateral values on borrowing capacity, and thereby on real activity. Asset price contagion can also have more direct effects, e.g., through real wealth effects. All these mechanisms represent independent contagion channels which may interact during the development of a financial crisis. For instance, the default of a bank may trigger an avalanche of deposit withdrawals at other banks as well as a liquidity evaporation on the interbank market.

This paper focuses on credit inter-linkages. Credit contracts establish connections among banks on the interbank market, among firms and banks on the market for loans, among customers and suppliers in the market for trade credit. In other words, the credit market can be conceived of as a credit network in which nodes represent agents and links represent credit relationships. To complicate the picture further, banks are also connected to financial intermediaries in the so-called shadow banking system, while financial products such as credit default swaps establish financial ties between the seller of protection and the reference entity. The most influential example of network analysis applied to the propagation of distress in a credit network is the seminal paper by Allen and Gale (2001) on "financial contagion", which has triggered a significant body of work, both on interbank credit (Freixas et al., 2000; Furfine, 2003; Boss et al., 2004; Elsinger et al., 2006; Iori et al., 2006; Nier et al., 2007), as well as on bank-firm or firm-firm credit (Stiglitz and Greenwald, 2003; Boissay, 2006; Battiston et al., 2007).

To capture the interdependence among banks that arises from these network relationships, we model the evolution of the levels of financial robustness - meant as an equity ratio - of different agents as coupled stochastic processes, similar to Hull and White (2001). Much of the earlier literature was directed at valuing corporate liabilities. In contrast, our objective is to understand the interplay of financial interdependence among banks and financial acceleration in the emergence of systemic risk. We assume banks' equity levels (and thus the levels of financial robustness) are subject to idiosyncratic shocks, along the lines of previous works (Allen and Gale, 2001; Allen and Babus, 2009; Allen et al., 2011; Wagner, 2010; Castiglionesi and Navarro, 2010). Thus, banks have an incentive to engage in risk sharing by financing the liabilities of other banks. This reduces the fluctuations on the value of their asset portfolio and hence their failure probability and associated risk premia. To model this, we consider a set of banks in which balance sheets are interconnected in a network of liabilities, following the framework of Eisenberg and Noe (2001) and Shin (2008).

On the other hand, as we have noted, connections entail a negative effect, through contagion. Results on systemic risk can be sensitive to the exact way in which contagion is modeled. It is often assumed that the bankruptcy of one bank may cause the failure of all $k$ connected banks (Stiglitz, 2010; Castiglionesi and Navarro, 2010). In an alternative setting, banks are assumed to be funded by investors, which upon the observation of a signal - the news that at least one bank will fail decide whether to roll over the loans (Allen et al., 2011). If they do not roll over, then all banks have to liquidate. Notice that in either case the failure of one bank may trigger the failure of $k$ banks, and so systemic risk increases with the connectivity of that bank. It is clear however that the diversification of the portfolios of counterparties of the failing bank lowers the likelihood of their failure. Thus, it is important to take into account the fact that financial ties are conducive of financial damage only proportionately to their relative weight in the portfolio of counterparties. On the other hand, we need to recognize that negative effects from one bank to another can arise well before the level of bankruptcy is reached. For instance, the fear of failure for a bank intertwined with another bank that faces a high risk of failure will drive up the interest rate which the former has to pay.

Because our model is dynamic and in continuous time, it allows us to consider the effect of variations in financial robustness from one bank to the others, rather than focusing solely on bankruptcy events. Moreover, in our model, the reason why we obtain an increase in the failure probability as connectivity increases is more subtle than in earlier contagion models and emerges from a milder assumption. We assume that counterparties of a bank are able to observe 
quarterly variations in its financial robustness (or some proxy) and when this variations are more negative than "normal", they take actions which penalize the bank. What is perceived as normal by the counterparties is the typical magnitude of the fluctuations of bank's robustness, as measured by its standard deviation $\sigma$ (in the absence of previous penalizations). When counterparties react, this translates in an immediate, further, drop in the bank's financial robustness by a magnitude $\alpha$. This assumption is meant to capture the adverse effect that may arise, for instance, because those providing credit increase the interest rate charged (Bernanke et al., 1999). Another situation is when some short-term creditors decide to hoard liquidity and not to roll over their funds, forcing the agent to fire-sell some assets in order to pay back the debts (Brunnermeier, 2008). In OTC derivative contracts, a drop in the level of financial robustness can trigger a collateral margin call, so the agent is forced to quickly free up some capital in order to honor the call. All these situations entail an additional cost for the agent, and thus a further reduction of its financial robustness.

The ratio $\alpha / \sigma$ between the amplitude of the adverse effect and the fluctuation amplitude plays a crucial role. It is clear that if $\alpha \ll \sigma$, then the penalization does not affect the probability of a further penalization, because the probability of a penalization is dominated by the random shocks. In contrast, if $\alpha>\sigma$, then it is likely that in the next time step the counterparties will observe another variation more negative than normal, and will react again. This chain of events may continue for several quarters in a row. We call trend reinforcement this particular form of persistence of financial distress. Notice that because of the random fluctuations, the chain will break as soon as the bank is hit by a shock positive and large enough to overcome the effect of the previous penalization. It follows that, as the ratio $\alpha / \sigma$ increases, once the bank has been caught in the trend reinforcement, the probability to escape decreases and the probability to default increases.

Since, we exclude by assumption systematic shocks to the industry, then shocks on financial robustness of agents come solely from the idiosyncratic returns on their projects. This implies that when agents engage in risk sharing with other $k$ agents, the magnitude of the typical fluctuations scales down as $\sigma / \sqrt{k}$, under the assumptions that shocks are uncorrelated. Counterparties of each agent are assumed to observe this and to base their perception of "normality" on the distribution of shocks in that environment.

Thus, when penalization by counterparties is ruled out by assumption, then with smaller shocks it takes longer for the robustness to hit the default barrier. In this case, diversification is beneficial. In contrast, when penalization is possible, and if the magnitude $\alpha$ of the penalization is constant (or scales down with $k$ more slowly than $\sigma$ ), then it is clear that with higher diversification the trend reinforcement is now more likely to occur and the default is reached faster. Notice that, ex ante, the probability to incur in a penalization in the first place is completely independent of $k$, because the level of "normality" is relative to the shock amplitude. Therefore, the model does not contain among the hypotheses any cost or disadvantage of diversification.

Notice that the case in which $\alpha$ remains constant with $k$ is a common situation that can arise in the presence of indivisibilities and fixed costs in the transactions. For instance, if the counterparties increase the interest rate charged to the agent by a fixed amount. Similarly, if an OTC derivative counterparty requires a fixed amount of additional collateral.

In general, there is also another effect which makes diversification even more problematic. As agents get connected in a network, their portfolios become more similar and shocks hitting the portfolio values become more and more correlated (Allen et al., 2011). The correlation among shocks on portfolios results in correlation across agents' levels of financial robustness so their trajectories tend over time to remain close to each other. This implies that, conditional on one agent reaching the default level, it is more likely that several other agents are also about to default. In other words, with higher diversification, systemic defaults, conditional to having at least one default are more probable. ${ }^{1}$ Notice that this holds in general as it follows solely from the correlation. In the presence of financial acceleration, the effect is stronger because it is more likely that a shared negative shock amplifies an already negative trend. ${ }^{2}$

What we have described so far is an evolution of financial robustness which one can think of as occurring at the time scale of months. Every quarter, balance sheets are revealed, ratings of firms are revised and financial ratios of those holding their liabilities move up or down. However, in the acute phase of the unraveling of a crisis, some agents default on their obligations, some assets are written down and this may cause other agents to default, thus triggering a cascade which evolves at the time scale of days and is usually mitigated by the intervention of the central bank. Accordingly, in the second part of this paper, we analyze the dynamics of failure cascades. There is a large and growing literature on failure cascades, since the earlier work of Allen and Gale (2001) and Stiglitz and Greenwald (2003). We follow an approach that is similar to the fictitious sequential default introduced by Eisenberg and Noe (2001) and in line with Gai and Kapadia (2010). When an agent defaults, the loss for its counterparties is assumed to be proportional to their relative exposure to it. A fraction of this loss can be in principle recovered by selling debtor's assets, but not in the very short term. Under some regularity assumptions on the structure of the network and the independence of defaults, it is possible to compute the expected size of the default cascade. We can then show that the probability of large cascades in the system is also not monotonically decreasing with the level of diversification. However, unlike other default cascade models, here cascades are investigated in combination with the slower dynamics of financial robustness. In summary, our results indicate that

\footnotetext{
${ }^{1}$ The joint default probability, conditional to the occurrence of one or more default, should not be confused with the probability of default we have discussed in the earlier paragraphs.

2 Even in a static context of two connected agents, Wagner (2010) finds a similar adverse effect of diversification, resulting from the assumption that the "loss is larger when agents have to liquidate at the same time". In contrast, in our model, we do not consider the cost of the crisis. High diversification is not desirable simply because it leads to larger but also more frequent crises.
} 
when the level of risk diversification of agents is such that they are embedded in a densely connected network of mutual liabilities, the financial system becomes very unstable and prone to frequent and large crises.

The paper is organized as follows. In Section 2 we present the model of the evolution of financial robustness and the results on the probability of individual defaults. Section 3 describes the dynamics of default cascade and the probability of systemic defaults. Section 4 concludes.

\section{A dynamic model of financial robustness}

In this section we introduce a minimalistic dynamic model describing the evolution of the financial robustness of agents connected in a network of credit relations. Similar to Hull and White (2001) and Zhou (1997), financial robustness of agent $i$ plays here the role of an indicator of the agent's creditworthiness or distance to default. We think of it as an equity to total asset ratio and we denote it as $\eta_{i} \in[0,1]$, with $\eta_{i}=0$ indicating bankruptcy. In line with the literature on the distance to default, we formulate the model in terms of a system of stochastic differential equations (SDEs) describing the evolution in time of all the $\eta_{i}$. The interest of this approach comes from the possibility of applying well-known standard methods to compute the expected first passage time at 0 and thus the probability of default of an agent taking into account the dynamics of the other agents. ${ }^{3}$

\subsection{Financial acceleration}

Our aim is to develop a model that captures at the same time two important mechanisms present in a financial network. The first mechanism, which we call financial acceleration, refers to the fact that current variations in equity depend on past variations in equity itself. This occurs in at least two scenarios. In the first one (see e.g., in Morris and Shin, 2008), an agent is hit by a shock due to a loss of market value of some securities in her portfolio. If such shock is large enough, so that some of her creditors claim their funds back, the agent is forced to liquidate at least part of her portfolio to reimburse debt. If the securities are sold below the market price, the asset side of the balance sheet decreases more than the liability side and the agent's fragility - i.e. leverage - goes up unintentionally. This situation can lead to a spiral of losses and decreasing robustness. At a macro-economic level, this corresponds to the procyclical nature of leverage (Morris and Shin, 2008; Brunnermeier, 2008; Brunnermeier and Pederson, 2009). A second scenario is the one in which when the agent is hit by a shock at time $t$, her creditors make credit conditions harsher at $t+1$. Indeed, it is well documented that lenders ask a higher external finance premium when the borrowers' financial conditions worsen (Bernanke et al., 1999). Stricter credit conditions can be considered an additional shock hitting the borrower in $t+1$.

If we translate this idea in terms of SDE (Gardiner, 2004; Oksendal and Karsten, 1998), the financial acceleration can be seen as a jump term that depends on the past realizations of robustness itself. For the sake of simplicity, we restrict here the dependence at time $t$ on the past only to one point in time $t-\tau$ (with $\tau>0$ ). We have in mind a situation in which the robustness of an agent evolves daily, due to the change in market value of its assets. However, typically, the conditional shock associated with financial acceleration occurs each quarter, when the financial situation of the agent is made public and, for instance, the rating of the agent is revised downwards.

Therefore, we assume that a jump possibly occurs only at each discrete time $t \in \mathcal{N}$, depending on the past realization of robustness at the discrete time $t-\tau$ (with $\tau \in \mathcal{N}$ ). We then have the following jump-diffusion equation:

$$
d \eta_{i}=\sigma d \xi_{i}+h(t) d N
$$

with

$$
d N=\sum_{t \in \mathcal{N}} \delta\left(t^{\prime}-t\right)
$$

In the above equations $\sigma$ is the standard deviation of the idiosyncratic shocks hitting agent $i$, $d \xi_{i}$ denotes the Wiener process and $\delta\left(t^{\prime}-t\right)$ is the Dirac delta-function. ${ }^{4}$

We further assume that $i$ 's partners react whenever there is a decrease of $i$ 's robustness perceived as "atypical", given the current market conditions. In other words, the penalty depends only on the change in robustness and not on its absolute level. ${ }^{5}$ Thus, we define

$$
h(t)= \begin{cases}-\alpha & \text { if } \eta_{i}(t-d t)-\eta_{i}(t-d t-\tau)<-\epsilon \sigma_{\tau}, \\ 0 & \text { otherwise }\end{cases}
$$

\footnotetext{
${ }^{3}$ Indeed, if we were to focus exclusively on the present discounted value of the bankruptcy costs, we would only need to study the value of the moment generating function of the first passage time.

${ }^{4}$ By definition then, the integral $\int_{t-d t}^{t+d t} \delta_{t^{\prime}, t} d t^{\prime}$ equals 1 on any interval including $t$ and equals 0 on any interval not including $t$. This means that in Eq. (2) the jump contributes to the variation of $\eta_{i}$ only at discrete times.

${ }^{5}$ There are of course other ways to model how partners react to the signal of distress coming from $i$, resulting in various functional forms of $h$. Another option would be for instance to assume that a penalty is triggered whenever the firm robustness goes below a certain threshold. This however, requires to make a choice about the proportion of firms that are initially born above or below such a threshold, thus complicating the scenario. A general investigation of the impact of the functional form of $h$ would be certainly of interest but is beyond the scope of the present work.
} 
where $\sigma_{\tau}$ is the standard deviation of the Gaussian shocks accumulated over a time delay $\tau, \sigma_{\tau}=\left(E\left[\left(\int_{0}^{\tau} d \xi_{i}\right)^{2}\right]\right)^{1 / 2}=\sigma \sqrt{\tau}$. The parameters $\epsilon$ and $\alpha$ represent, respectively, the sensitivity of counterparties' reaction, and the amplitude of the effect of such a reaction on $i$ 's robustness. The definition above implies that the counterparties of $i$ react only when an adverse shock to $i$ is large enough to cause a negative variation of robustness that exceeds, in absolute value, $\epsilon$ times the standard deviation of the shocks, $\sigma_{\tau}$-in other words, the shock is perceived as "atypical". When the counterparties do react, the reaction act as a penalty causing a decrease of magnitude $\alpha$ in $i$ 's robustness. ${ }^{6}$ Indeed, whether creditors refuse to roll over debt, or they increase the interest rate charged to $i$, the overall effect is an additional cost and thus a further decrease of $i$ 's robustness. Thus, Eq. (3) is a simple way to model, in a reduced form, the chain of events described earlier in the two scenarios where financial acceleration is at work. We will refer to the repercussion for $i$ a magnitude $\alpha$, as penalty and/or as conditional shock.

A large financial robustness implies also a low leverage. Agents have no incentive to keep an indefinitely large equity ratio. Thus, we assume that robustness does not exceed an upper reflective barrier at $\eta_{\max }$. Bankruptcy occurs when robustness falls below a given threshold $\eta_{\min }$, modeled, as usually done, as a lower absorbing barrier. Thus, over time the agent goes bankrupt when robustness hits the lower barrier and is replaced by a new agent, with a new initial value of robustness $\eta_{0}$. The time to default depends on the value of $\eta_{0}$, where the process starts, relative to the extremes of the

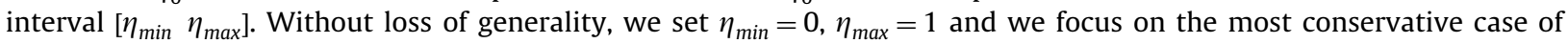
$\eta_{0}=1$.

Notice that Eq. (3) is likely to underestimate the level of acceleration that occurs in a real situation. We could expect that when agent $i$ 's robustness gets closer to the default threshold, the reaction of the partners, and its effect, increases in amplitude, and thus that $\alpha$ is a function of $\eta$, with $\alpha^{\prime}(\eta)<0$. For the sake of simplicity, we assume $\alpha$ constant, which is a conservative scenario. In any case, what really matters is the ratio between amplitude and sensitivity as we discuss below.

In the following, we restrict to the case $\tau=1$, which we interpret as conditional shocks occurring only at quarters. We thus have $\sigma_{\tau}=\sigma \sqrt{\tau}=\sigma$. Eq. (3) implies that if $h(t)=-\alpha$ and $\alpha>\epsilon \sigma$, then, in the next quarter, the counterparties of $i$ will observe again a variation of robustness $\eta_{i}$ that triggers their reaction, unless the continuous time shocks accumulated during the interval $[t, t+1]$ is positive and large enough to compensate for it. Thus, the larger the ratio $\alpha /(\epsilon \sigma)$ between the amplitude of the adverse effect and the sensitivity of the counterparties, the more frequently a negative variation in robustness will be followed by another negative variation. We refer to this effect as trend reinforcement, which is a specific form of amplification of financial distress. In particular, for higher values of the ratio $\alpha /(\epsilon \sigma)$, the expected time to default of the agent is shorter.

To see this more formally, we first note that in Eq. (3), the value of $h$ at time $t$ is a stochastic variable. Denote with $q$ the probability that $h(t)=-\alpha$. There are two cases in which the event $h(t)=-\alpha$ occurs. If $h(t-1)=0$, then the shock accumulated in $[t-1-d t, t-d t]$ has to be large enough (and negative). If, instead, $h(t-1)=-\alpha$, then a smaller shock is sufficient to imply $h(t)=-\alpha .^{7}$ This means that there is a positive feedback increasing the chance that $h(t)=-\alpha$ in course of time, up to a certain level. We can then compute the value of $q$ in equilibrium as shown in the following proposition (see Proof of Proposition 3).

Proposition 1. Consider the evolution of robustness of Eq. (1) in the presence of the financial acceleration defined by Eq. (3). Then, over time the steady-state probability $q$ that $h=-\alpha$ is

$$
q=\frac{\Phi(-\epsilon)}{1-\Phi\left(\frac{\alpha}{\sigma}-\epsilon\right)+\Phi(-\epsilon)}
$$

where $\Phi$ is the Gaussian cumulative distribution.

In equilibrium, the expected value of $h$ over time is of course $-\alpha q$, which means that the average effect of the trend reinforcement jumps is equivalent to a negative drift. This drift is stronger, the larger the $\alpha$ is with respect to $\epsilon \sigma$.

In the following, we are interested in the expected value of the first passage time of the system. In this respect, the average effect of the jump is well approximated by the effect of a constant drift. ${ }^{8}$ In contrast with a model with a timedelay dependent jumps, a model with constant drift is analytically tractable. Therefore, based on their equivalence from the point of view of expected first passage time, from now on we model the financial acceleration simply as a constant drift term and rewrite Eq. (1) as follows:

$$
d \eta_{i}=-\alpha q(\sigma, \alpha, \epsilon) d t+\sigma d \xi_{i}
$$

As the intuition suggests, the stronger the acceleration, the sooner the firms reach the default threshold, as stated in the following proposition.

\footnotetext{
${ }^{6}$ Notice that in the definition above, we want the counterparties to compare the current robustness to the one at the previous quarter, right before the possible occurrence of the penalty at the previous quarter. This is the reason of the $t-d t$ and $t-\tau-d t$.

7 Notice that in Eq. (3) the value of $h(t)$ depends on the realizations of the Wiener process in the interval [t-dt-1,t-dt] and it is independent of the realizations of the Wiener process in the interval of $[t-d t, t+1-d t]$.

${ }^{8}$ See in Appendix the numerical simulations supporting this claim.
} 
Proposition 2. Consider the evolution of robustness of $E q$. (5), with initial condition $\eta_{i}(0)=1$, in the presence of the financial acceleration defined by Eq. (3). Then, the probability of default increases monotonically with the intensity $\alpha$ of the financial acceleration.

\subsection{Interdependence}

A priori it is not clear at all how the default probability is affected when agents get connected in a network of contracts. Thus, interdependence is the second mechanism we want to include in the model, namely the fact that the financial robustness of an agent is affected by variations in the robustness of agents connected to her by some contracts. In line with the modeling framework introduced by Eisenberg and Noe (2001) and further developed in Shin (2008), we consider a set of $n$ agents connected in a network of obligations. This is described by a liability matrix $L$, where $L_{i j}$ is the obligation of $i$ to $j$ and $\left[L_{1 i}, \ldots, L_{n i}\right]$ is the portfolio of $i$. For our purposes, we are interested in the exposure matrix $W$, where $W_{i j}=L_{j i} / \sum_{k} L_{k i}$, represents the weight of the obligation of $j$ to $i$ relative to the whole portfolio of $i$. Precisely, $W_{i j}$ measures the relative financial exposure of $i$ to $j$, since this is the fraction of $i$ portfolio that, at most, is at risk in case $j$ becomes insolvent. It follows that $W$ is a row-stochastic matrix since the relative exposures of each agent to the others must sum up to one, $\forall i \sum_{j} W_{i j}=1$.

Formally, the relations in the network are represented by a graph $G=(\mathcal{V}, \mathcal{E})$, where $\mathcal{V}$ is a set of nodes representing the agents and $\mathcal{E}$ is a set of directed edges representing the contracts (for an introduction to network theory, see, e.g., Jackson, 2008; Goyal, 2007). The graph is associated with an adjacency matrix $A$ where $A_{i j}=0$ if there is no edge from $i$ to $j$ and $A_{i j}=1$ if there is an edge from $i$ to $j$. For the sake of convenience in matrix operations, we adopt the convention that the edge $(i j)$ means that agent $i$ owns liabilities of $j$. The exposure matrix $W$ corresponds to a weighted adjacency matrix with $W_{i j}>0$ iff $A_{i j}=1$. The out-degree of agent $i$ is the number $1 \leq k_{i} \leq n-1$ of out-going edges of agent $i$ and represents the number of counterparties or neighbors to which agent $i$ is exposed.

In the following, we will characterize the systemic risk of the credit network for varying levels of risk diversification. We are interested in the situation in which the graph is connected (i.e. there is some path from any agent to any other agent) because this means that every agent can potentially be reached by external effects. The case in which the network is divided in disconnected clusters will be investigated in further studies. Further, we do not consider any inter-temporal optimization of the portfolios. On the contrary, we assume that the exposures in the portfolio of each agent are approximately balanced, i.e. $W_{i j} \cong 1 / k_{i}$. This rather stylized assumption, besides simplifying the analysis, has however the advantage of implying a conservative scenario in which risk sharing in the system is always maximized. One can expect that results on the emergence of systemic risk should then hold a fortiori for networks where some agents are unequally exposed to some others.

Since portfolios are equally weighted, the degree of the agent $i$ is a rough measure of her risk diversification level. The ratio of the number of existing edges among agents, denoted as $\ell$, over the number of all possible edges, $n(n-1)$, is the density $d$ of the network, $d=\ell /(n(n-1))$. It represents the fraction of possible pairs of agents that are involved in a financial contract. A simple relation of proportionality holds between the average degree $k$ in the network and the density $d=k /(n-1) \cong k / n$ (for $n$ in the range we are interested in). Thus for a fixed number $n$ of agents, the density of the network is also a measure of the average risk diversification across the agents.

In the following, the analysis will be carried out for the case of a regular graph, i.e. when agents have the same level $k$ of diversification. ${ }^{9}$ Since $i$ 's assets include $j$ 's liabilities, their value must depend on the financial conditions of $j$. In particular, at least at first order, an increase of $j$ asset value will have a positive effect on $i$ portfolio, while an increase of $j$ liabilities (meant as cost of financing incurred by $j$ ) will have a negative effect. In other words, in a financial network, $i$ 's robustness must depend on $j$ 's robustness. However, the exact way this interdependence works is not simple and deriving a fully fledged law of motion of robustness would require to model also the decisions of agents regarding their capital structure and in particular whether and how to reduce their liability, given their expectations on the market conditions. Our aim here is much less ambitious and more pragmatic. We consider a first order linear dependence between the robustness of the agents in the networks and we investigate the implications of lower or higher risk diversification on the default probability. The results of this exercise provide interesting insights for future, more micro-founded works.

Let us start with the following law of motion of robustness in discrete time:

$$
\eta_{i}(t+1)=\sum_{j} W_{i j}\left(\eta_{j}(t)+\sigma \xi_{j}(t)\right)
$$

where we assume that robustness of each agent is subject to idiosyncratic identically distributed and normal shocks. Within a similar framework one could also account for the case of correlated shocks. For our purposes this case will suffice

\footnotetext{
${ }^{9}$ The empirical evidence on financial networks indicate the tendency to exhibit a core-periphery structure. The core alone is quite dense and not so heterogeneous in degree (Soramaki et al., 2007; Iori et al., 2008; Vitali et al., 2011). For this reason, the following analysis can be relevant to the core of a financial network, which typically is also where most of the total asset value resides.
} 
here. Since the liabilities of agent $j$ are held by other agents, the idiosyncratic shock hitting $j$ is shared with the connected agents proportionally to the relative weight of their financial exposure to $j .{ }^{10}$

Notice that the above formulation is similar in concept to the model of Allen et al. (2011) where each agent runs an independent project with a stochastic return and, in order to diversify their risk, agents invest in other agents' projects. As a result, each agent is affected by the linear combination of the shocks of her partners. However, differently from that model, here we do not look at the return, but at the robustness of the agents. Moreover, we depart from the modeling framework of both Allen et al. (2011) and Shin (2008) by moving to a fully dynamic setting in order to account for variations in the level of robustness, as opposed to considering only the default event.

The change in time of robustness ${ }^{11}$ is

$$
\eta_{i}(t+1)-\eta_{i}(t)=\sum_{j} W_{i j} \eta_{j}(t)-\eta_{i}(t)+\sigma \sum_{j} W_{i j} \xi_{j}(t)
$$

Passing now to the limit of continuous time we obtain the linear SDE system

$$
d \eta_{i}=\left[\sum_{j} W_{i j} \eta_{j}(t)-\eta_{i}(t)\right] d t+\sigma \sum_{j} W_{i j} d \xi_{j}
$$

where each $d \xi_{j}(t)$ denotes an independent Wiener process.

The benefit of an agent from being connected to several other agents is that the expected fluctuation on the robustness is decreased and thus the time to hit the default barrier is longer. This follows from the fact that the linear combination of $k$ uncorrelated Wiener processes is still a Wiener process, denoted as $d z_{i}=\sum_{j} W_{i j} d \xi_{j}$, with a standard deviation $\sigma_{z}$ smaller by a factor $1 / \sqrt{k}: \sigma_{z}=\sigma / \sqrt{k}$.

We thus obtain

$$
d \eta_{i}=\left(\bar{\eta}_{i}(t)-\eta_{i}(t)\right) d t+\sigma_{z} d z_{i}
$$

where $\bar{\eta}_{i}=\sum_{j} W_{i j} \eta_{j}$ is the average robustness of the neighbors of $i$. Notice that risk sharing and distress propagation are two faces of the same coin. By investing in others' liabilities each agent is sharing her own risk with them but also accepting to be affected by the variations, positive or negative, of the robustness of the others. In bad times, when someone in the neighborhood is badly hit by a shock, this means that the agent is exposed to a distress propagation.

\subsection{Acceleration in a network context}

When the agent $i$ is in a network of risk sharing relations, her creditors will observe now a standard deviation $\sigma_{z}$ in the fluctuations of its robustness that are due to shared random shocks. As before, in the case $\tau=1$, we assume

$$
h(t)= \begin{cases}-\alpha & \text { if } \eta_{i}(t-d t)-\eta_{i}(t-d t-1)<-\epsilon \sigma_{z, 1}, \\ 0 & \text { otherwise }\end{cases}
$$

where $\sigma_{z, 1}=\sigma_{z} \sqrt{1}=\sigma / \sqrt{k}$ is the standard deviation of the Gaussian shocks, accumulated over a time period of length 1 , if agent $i$ engages in risk sharing with $k$ other agents. In other words, $i$ 's counterparties react according to the current level of fluctuation amplitude. However, it is important to emphasize that the probability that $i$ incurs in a penalty is exactly the same as in the previous case without risk sharing (as long as there was no penalty at the previous quarter). ${ }^{12}$

Notice also that, counterparties' reaction on $i$ has remained of the same amplitude, $\alpha$. In other words, the magnitude of the penalty has not changed when moving to the network context. Clearly, if $\alpha$ would also change into $\alpha / \sqrt{k}$, then the trend reinforcement would not step in for increasing $k$. In this case, risk sharing has the usual positive impact on the probability of default.

The interesting case is when the magnitude of the penalty and the magnitude of the fluctuations do not scale the same way with the number of neighbors. Here, we focus on the case in which $\alpha$ remains constant with $k$, a situation that can arise from indivisibilities and fixed costs in the transactions. For instance, if each of the counterparties of $i$ increases the interest rate charged to the agent by a fixed amount, the effect for $i$ is simply proportional to her total debt and is not

\footnotetext{
${ }^{10}$ In case $j$ is not able to meet her obligation, $W_{i j}$ is the relative loss of $i$ with respect to her initial investment, thus $W_{i j}$ should be a good proxy of how much the variation of robustness of $i$ are affected by the robustness of $j$. Notice that this may differ from the loss with respect to the value of the assets at a later point in time. However, if the returns on the various assets do not differ too much, or one considers periods of time that are not too long, $W_{i j}$ is a reasonable proxy of the relative impact on $i$ 's asset due to a change in robustness of $j$. Accordingly, the shock hitting agent $i$ affects $i$ herself only proportionally to the term $W_{i i}$.

11 Notice that Eq. (6) implies that the robustness of an agent is not the result of a simultaneous computation of robustness of all agents. Instead, it is affected by the change in robustness of the counterparties at the previous period. This is similar to what happens in the professional practice of financial rating. The rating of an institution is revised after the occurrence of events regarding the capital structure of the institution itself or the counterparties to which it is exposed.

12 The probability to trigger the reaction of the counterparties equals the probability that a (accumulated) shock is beyond a given threshold (see also proof of Proposition 1). This is simply the probability mass in the left tail of the probability distribution, $q_{0}=\Phi\left(0, \sigma_{z},-\epsilon \sigma_{z}\right)=\Phi(0, \sigma,-\epsilon \sigma)$, where $\Phi(\mu, \sigma, x)$ indicates the cumulative Gaussian distribution up to the value $x$. Because both the shocks and the level beyond which they trigger a penalty are rescaled by $1 / \sqrt{k}$, the probability of a penalty (conditional to no prior penalty) is unchanged.
} 

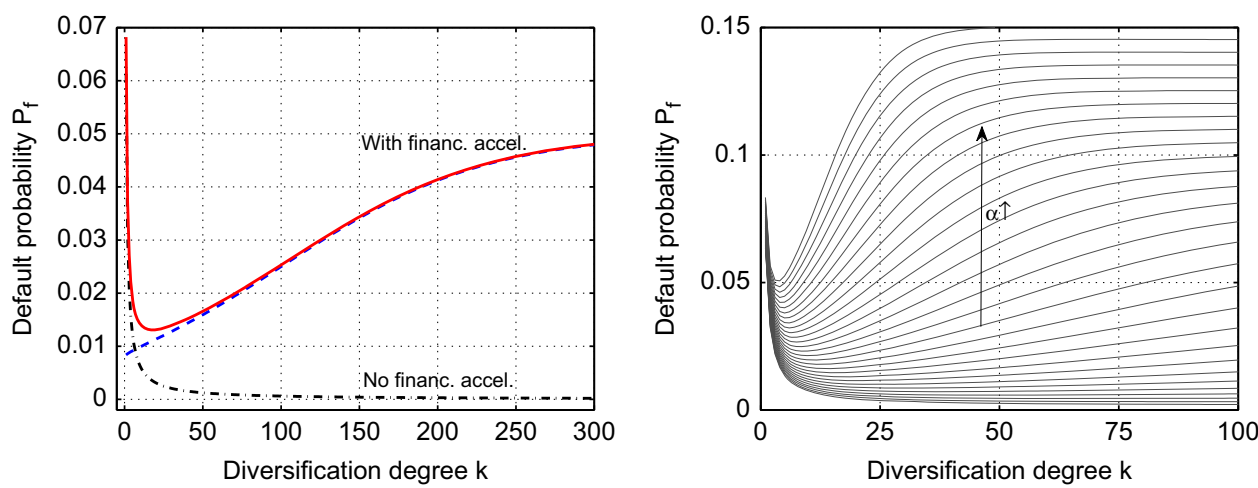

Fig. 1. Default probability $P_{f}$ as a function of the diversification degree $k$. (Left) Comparison of presence (solid line) and absence (dotted line) of the financial acceleration. The dot-dashed line represents the values of the term $\alpha q(k)$ in Eq. (11). Parameters setting: $\epsilon=1, \sigma=0.25, \alpha=0.055$. (Right) Illustration of dependence on $\alpha$. Parameters: $\epsilon=1, \sigma=0.25, \alpha$ varies in [0.01 0.15] in steps of 0.005 .

diminished by her level $k$ of diversification. The same holds for an OTC derivative contract if each counterparty requires a fixed amount of additional collateral after she observes the signal about $i$ 's robustness.

Proposition 1 holds again with $\sigma_{z}$ replacing $\sigma$, as well as the good approximation, in terms of expected first passage time, between the jump-diffusion model and the model with constant drift. We thus add the drift term $-\alpha q$ in Eq. (9) and we obtain the final formulation of our model

$$
d \eta_{i}(t)=\left(\bar{\eta}_{i}-\eta_{i}(t)-\alpha q\left(\sigma_{z}(k), \alpha, \epsilon\right)\right) d t+\sigma_{z} d z_{i}
$$

Notice that $q$ depends now also on the degree $k$ of diversification, via $\sigma_{z}$.

With $\sigma_{z}=\sigma / \sqrt{k}$, Proposition 1 implies now that the average effect of the trend reinforcement, $-\alpha q$, depends on the ratio between $\alpha$ and $\sigma / \sqrt{k}$. In particular, it increases with $k$ up to an asymptot, as shown in Fig. 1 (left, dashed curve). The curve means that, for small $k$, it is unlikely that an agent suffering a decrease in robustness will be penalized and will incur in a further decrease of robustness. However, as $k$ increases, $\sigma_{z}$ decreases and the fluctuation magnitude gets dominated by the magnitude of the effect of the penalty, $\alpha$. It becomes thus more and more likely that an agent suffering a decrease in robustness will be penalized further in the next quarter. Notice that the magnitude of the instantaneous effect of the penalty remains always $\alpha$. It is only its average effect to become stronger because it occurs more often.

In summary, an increasing level of diversification $k$ in the network is initially beneficial to the agents. Indeed, smaller fluctuations on asset portfolio values imply longer time to default. However, beyond a certain level of $k$, whenever an agent suffers a relatively large negative shock, the effect of trend reinforcement steps in.

A crucial point here is that, regardless the diversification level, the probability of occurrence of the first large negative shock is always the same, thus there is no ex ante disadvantage from a high diversification. However, with higher diversification the trend reinforcement is more frequent because of the different balance between penalty and fluctuations, implying a shorter time to default. This ambiguous effect of diversification is an unforeseen external effect of the reaction of $i$ 's counterparties which eventually hits back all the agents.

\subsection{The interpretation of acceleration in a network context}

Our interpretation of trend reinforcement in Eq. (10) starts from the assumption that each agent reacts to a negative change in the robustness of a partner belonging to the same neighborhood generated by idiosyncratic Gaussian shocks to all the nodes in the neighborhood only when the negative change exceeds, in absolute value, a certain threshold, i.e. only when the performance of the agent becomes poor. The performance is poor when the change is not only negative but negative beyond normal, with a normal or acceptable loss to be defined as one standard deviation from the mean of the idiosyncratic shock. If the loss is contained within the normal/acceptable level (that is if it is smaller than the threshold) there will be no reaction (and punishment) on the part of the neighbors.

The acceptable or "normal" loss, in turn, is decreasing with density. In fact, the idiosyncratic shock to each agent is drawn from a (time invariant) distribution with variance $\sigma$. Each shock is then spread to the neighborhood through credit inter-linkages, so that each partner is hit only by a fraction of the shock itself. The normal loss therefore is $\sigma / \sqrt{k}$, hence it is decreasing with density. If the network becomes more dense (i.e. if the network is characterized by higher $k$ ) the riskiness of an agent for each partner will become smaller. This is straightforward and descends from the fact that each agent is discharging part of the idiosyncratic shock on the partners: the more numerous is the neighborhood, the smaller the fraction of the shock to a partner which reaches the other partners. When an agent is hit by a negative shock and records an abnormal loss, i.e. a decrease of robustness beyond the threshold, she sends a signal of "poor" performance to her partners. If the average partner is relatively diversified (i.e. the network is characterized by a high $k$ ), the performance is considered "poor" when the loss reaches a magnitude smaller than the one which triggers the rating of poor performance 
in case the average partner has a more concentrated portfolio (low $k$ ). So far therefore, we have the following sequence: the threshold is an increasing function of the riskiness of each agent as measured by $\sigma / \sqrt{k}$ and the riskiness in turn is decreasing with density.

This interpretation can be complemented by the following arguments:

(1) We can assume that more diversified agents will take on higher levels of debt financing. This conjecture is based on a notion of riskiness different from that of the previous paragraph and captured by $\sigma / \sqrt{k}$. This new notion of riskiness is based on prospective financial fragility: an agent becomes more risky (more financially fragile in the future) in the eyes of her neighbors when she becomes more diversified. This is a possible complementary cause of the negative relationship between the threshold and connectivity $k$. We have no specific reference to support this conjecture. It is empirically plausible, however, that a more dense network of credit inter-linkages makes the market for loans thicker and credit more easily accessible to firms, thereby boosting leverage. For instance one may argue that the practice of multiple lending relationships among a firm and a certain number of banks makes easier for firms to get into debt and therefore there should be a relationship from connectivity to leverage and financial fragility. These issues have been explored by Ongena and Smith (2000) who conclude that there is a positive correlation between financial fragility and the practice of multiple lending relationship.

(2) We can assume that the signal implied by poor results at one firm has stronger impact for the likelihood that others are in trouble if the degree of interconnectedness is greater. This follows from the simple fact that there are more firms connected to the agent having a poor performance in the case of a high $k$.

Both factors will lead neighboring firms to respond more sensitively to "poor" reported results by any single firm with whom they do business.

\subsection{The probability of individual defaults}

We now analyze the impact of interdependence and financial acceleration on the probability of bankruptcy. In a nutshell, we find (not surprisingly) that, in the absence of financial acceleration, the diversification of individual risk always yields a reduction of individual default probability. In contrast, as soon as we include the financial acceleration, this is not necessarily true. Financial acceleration, as shown by Proposition 1, generates an average negative drift in the stochastic trajectory of the robustness that more than offsets the positive effect of diversification. As we have noted, the probability of individual bankruptcy can be investigated in terms of the first passage time of the SDE. For Eq. (11), it is possible to compute analytically the mean passage time of robustness at 0 and hence the probability of bankruptcy, to ascertain more precisely how it is affected by diversification.

The probability $P_{f}$ that, at any given time $t$, an agent goes bankrupt is the expected frequency with which, over time, the robustness of the agent hits the bankruptcy threshold. Since default frequency is the inverse of the time between successive events, the probability of bankruptcy can be measured as the inverse of the mean first passage time, $T_{f}$, of the stochastic process describing the evolution of the robustness, $P_{f}=1 / T_{f}$. The mean first passage time of a stochastic process in the presence of both a reflective and an absorbing barrier can be computed with standard approaches (Gardiner, 2004), leading to the following proposition.

Proposition 3. Consider a regular random network of financial agents whose robustness follows Eq. (11), with $q(\sigma / \sqrt{k}, \alpha, \epsilon)$ given by Eq. (4). Denote the average probability of bankruptcy of an individual agent as $P_{f .}$ Then

1. In the absence of financial acceleration $(h=0, \forall t), P_{f}$ decreases with the degree $k$ of risk diversification as $P_{f}(k)=\sigma^{2} / k$.

2. In the presence of financial acceleration $(h<0 \exists t)$

(a) $P_{f}$ depends on $k$ as follows:

$$
P_{f}=\frac{\alpha q(k)}{1+\frac{\sigma^{2}}{2 k \alpha q(k)}\left(\exp \left(-2 k_{i} \alpha q(k) / \sigma^{2}\right)-1\right)}
$$

3. As function of $k, P_{f}(k)$ is not, in general, decreasing monotonically and it has a unique minimum for several parameter settings.

The first result is due to the fact that in the absence of financial acceleration the evolution of the robustness can be approximated with a Brownian motion. In this case, the mean first passage time through an absorptive barrier is inversely proportional to the variance of the random steps. This implies the intuitive result that the probability of bankruptcy decreases with the average number of counterparties. In other words, the credit network is more stable the larger is the number of contracts among agents. These results are in line with the classical result of Allen and Gale (2001).

Concerning the second and third result, the dashed curve in Fig. 1 (left) represents the absolute value of the drift, $\alpha q$. As discussed in the previous section, for small $k$, fluctuations of $\eta$ are comparable in magnitude with $\alpha$, thus the trend reinforcement occurs only seldom and, on average, the drift is small. As $k$ increases, agents tend to remain trapped in a sequence of trend reinforcements, which corresponds, on average, to a stronger drift. As shown in Fig. 1 (right) the probability of default does not 
grow indefinitely with $k$, but tends asymptotically to a finite value that depends on $\alpha$. As a consequence, in the presence of acceleration, the probability of default, $P_{f}$, turns out to have a minimum as a function of $k$, in a whole range of values of $\alpha$ and $\sigma$. This implies that risk diversification becomes at some point counterproductive and increases the probability of default.

The models in which the mathematical structures exhibit "convexities", in the relevant sense, diversification will naturally improve performance. Trend reinforcement (acceleration) can be viewed as introducing a natural non-convexity. Thus, while this result is at odds with the widespread presumption in favor of diversification, it is consistent with several recent works (Wagner, 2010; Castiglionesi and Navarro, 2010; Stiglitz, 2010, forthcoming) which show that full diversification is not optimal.

\subsubsection{Positive feedbacks versus fluctuations}

In our model, financial acceleration is nothing else than a positive feedback loop that, let alone, would eventually lead the agents towards default. Fluctuations have a twofold effect. On the one hand they can contribute to the path of the agent towards default. On the other hand they can cut the loop of the positive feedback. Excessive risk sharing, by decreasing the fluctuations, eventually prevents to cut the loop of the positive feedback, leaving the agent exposed to the effect of acceleration alone.

Of course this effect holds only if the magnitude of the penalty, $\alpha$, does not scale down with $k$, or at least if it does so slower than the magnitude of the fluctuations, $\sigma / \sqrt{k}$. We have assumed $\alpha$ constant, which is an interesting and not uncommon case, as discusses earlier.

The model essentially shows, analytically and in a relatively simple framework, that in the presence of a potential instability towards default, fluctuations do not necessarily increase instability. Instead fluctuations can help to mitigate the instability by cutting the loop of the positive feedback.

\subsection{Coupled dynamics and systemic defaults}

Since the evolution of robustness in Eq. (11) is described by a linear system, the distribution $p\left(\eta_{i}(t)\right)$ of the values of robustness of a given agent $i$ across different realizations of the process is Gaussian (Gardiner, 2004). We cannot say the same for the probability distribution $p(\eta, t)$ of the values of robustness across agents in a given realization at a given time. In general, the two distributions may not be the same. The latter is the one we need in order to estimate the probability that, conditional to the default of one agent, a certain number of other agents fail too.

However, via the Ito lemma one can derive a scalar SDE for the variance $v(t)=\sum_{j}\left(\eta_{j}(t)-\bar{\eta}(t)\right)^{2} / n$ of the robustness across the agents at any time. ${ }^{13}$ Under some regularity conditions (i.e. the graph has to be primitive, Seneta, 2006), the expected value of the variance tends exponentially fast over time to the value $\left(\sigma^{2} / k\right)-\left(\sigma^{2} / n\right)$. Therefore, apart for the correction term $\sigma^{2} / n$, the variance of the robustness across agents coincides with the variance of the shocks at the level of individual agents. In other words, over time, after a transient phase, the trajectories of $\eta_{i}(t)$ at the different agents evolve staying within a sort of bundle of constant width. This is due to the mean reversion term, $\sum_{j} W_{i j} \eta_{j}-\eta_{i}$, in the dynamics. The width of the bundle decreases as $k$ increases, so that trajectories of robustness remain more and more close to each other. This increase of correlation with the diversification has two implications concerning the systemic defaults.

The first implication is that the probability that several agents fail, conditional to the default of at least one of them, grows with $k$. The effect of correlation on systemic defaults have been investigated in Allen et al. (2011) where, connectivity $k$ is fixed but correlation depends on the network architecture. Notice that, in our case, even if the individual default probability $P_{f}$ were constant with $k$, the correlation implies that the probability of multiple defaults grows with the level $k$ of diversification, provided of course that the network satisfies the regularity conditions mentioned above.

The second implication is that, when the default of an agent has external effects on the connected agents (effect which we have not taken into account so far), we can expect the probability of multiple defaults to grow even faster with the level $k$ of diversification. The reason is that if the default of one agent decreases the financial robustness of the creditors, since these must be also close to the default barrier (due to the high correlation to the defaulted one) they are now even more likely to fail. In order to quantify this effect, in the next section we introduce in the model a default cascade dynamics.

\section{Bankruptcy cascades}

In the dynamics we have introduced in the previous section, the change in robustness of an agent is affected at any point in time by the robustness of the counterparties. In this section, we introduce a different dynamics, in which the default of an agent has a negative external effect on the robustness of its counterparties. If some of these counterparties, in turn, default on their own counterparties, this can trigger a cascade of defaults, a phenomenon often referred to as domino effect. Our cascade dynamics, although stylized, is conceptually very similar to the well established framework of Eisenberg and Noe (2001). Formally it turns out to be similar also to the dynamics introduced more recently in Gai and Kapadia (2010), although there some important differences in the methodology applied to solve for the size of the

\footnotetext{
13 This is a standard application of the Ito Lemma (Oksendal and Karsten, 1998). A proof is available from the authors upon request.
} 
cascade. ${ }^{14}$ Further, our objective is to assess how the probability of occurrence of large cascades is affected by the average level of diversification across the agents, in particular when cascades are considered in combination with the dynamics of robustness subject to acceleration.

A formal way to deal with the effects that payment default has on the cash flow of the counterparties is the fictitious default sequence introduced by Eisenberg and Noe (2001) with the objective of estimating the value of claims when agents are connected in networks of obligations containing circular chains (e.g., A lends to B, B lends to C, C lends to A). The idea is that the default of an agent decreases the value of the assets of each counterparty in the interbank market, and if the asset value becomes smaller than the liabilities, the counterparty in turn defaults, thus triggering a cascade. As shown in Gai and Kapadia (2010), when exposures among agents are equally weighted and thus risk sharing is maximized, only two variables matter in the cascade dynamics. The first is the fraction of defaulting counterparties of each bank and the second is the default threshold, which varies across banks and depends on the initial value of assets and liabilities of the bank.

Here, we assume that, as a result of the default of a counterparty $j$, the robustness $\eta_{i}$ of agent $i$ decreases by an amount proportional to the relative exposure $W_{i j}$, consistently with the network of liabilities described in Section 2.2. Formally the dynamics in discrete time reads as follows:

$$
\eta_{i}(\tau)=\eta_{i}(0)-\frac{a}{k} \sum_{j} W_{i j} \chi_{j}(\tau)
$$

where the function $\chi_{j}(\tau)$ indicates if the agent $j$ has defaulted at any previous time $1, \ldots, \tau$

$$
\chi_{j}(\tau)= \begin{cases}1 & \text { if } \eta_{j}\left(\tau^{\prime}\right)<0, \exists \tau^{\prime} \leq \tau, \\ 0 & \text { else }\end{cases}
$$

For convenience with respect to what comes in the following, we have denoted the time variable as $\tau$, so to distinguish it from the variable $t$ used in the dynamics of Eq. (11). The parameter $a$ determines the extent of the loss caused by the default of $j$ to its counterparties. In the hypothesis that there is no asset recovery during the development of the cascade we set $a=1$. Notice that, if we assume also maximal risk sharing, i.e. $W_{i j}=1 / k_{i}$, the dynamics above implies a decrease of robustness proportional to the fraction of defaulting counterparties of $i$, exactly as in Gai and Kapadia (2010). One difference is that here the default threshold is fixed at 0 , while the initial level of robustness differs across agents.

The cascading process just described is deterministic and terminates for sure after a finite number of steps smaller than the number of agents (Kleinberg, 2007). In the final state, a certain fraction $s$ of the agents has defaulted.

Notice that this approach is conservative in the sense that the aggregate loss caused by each defaulting agent to her counterparties coincides with their exposures. This is unlike other models of contagion in financial systems (Stiglitz, 2010), as well as models of innovation diffusion and disease contagion (Jackson and Rogers, 2007), where the aggregate loss increases with the number of contacts, because there is an independent probability of contagion associated to each link.

\subsection{The size of a cascade}

The number of defaults at the end of the cascade process can be computed under some simplifying hypotheses. The technique consists in deriving a recursive equation for the cumulative fraction of defaults in the system as a function of the cumulative fraction of defaults at the previous step. Solving for the stable fixed point of such equation yields the size of the cascade. $^{15}$

The recursive function depends of course on the initial distribution of robustness across agents. If agents have little robustness, the cascade tends to be larger because the default of some is more likely to cause the default of others. Based on the discussion in Section 2.6, we will assume in the following that the initial distribution of robustness when the cascade starts can be approximated by a Gaussian with variance $\sigma^{2} / k$. However, a similar computation could be carried out for other probability distributions of robustness. For our purposes, we fix the variance of individual shocks $\sigma^{2}$ and we focus on the diversification $k$ as a varying parameter. The result about the cascade size is stated in the following proposition.

Proposition 4. Consider the process of Eq. (13). Assume the network of firms is a regular random graph with degree $k$. Assume also the initial probability distribution of robustness is Gaussian with mean $m$ and variance $\sigma_{\eta}^{2}, p(\eta, \tau=0) \sim$ Gauss $\left(m, \sigma_{\eta}\right)$. Let $\Phi_{m, \sigma_{\eta}}$ denote the cumulative probability distribution of $\eta$ and $s_{0}$ denote the fraction of firms whose robustness is below zero at the beginning of the process. Then

1. The fraction $s$ of defaults at the end of the cascade process is the solution of the equation

$$
s=\max \left\{s_{0}, F\left(s, m, \sigma_{\eta}\right)\right\}
$$

\footnotetext{
${ }^{14}$ See also Stiglitz and Greenwald (2003) for an alternative model of bankruptcy cascades.

15 This method differs from the graph generating function approach used in Gai and Kapadia (2010).
} 

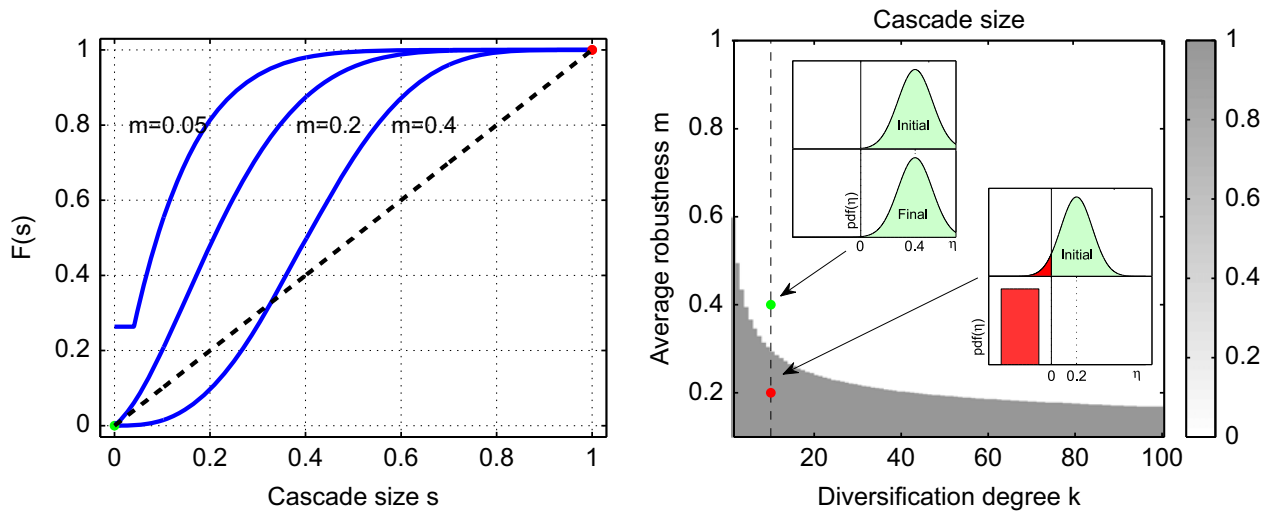

Fig. 2. (Left) Plot of R.H.S. of the fixed point Eq. (14) for the cascade size. Examples for $\sigma=0.25$ and $k=10$. For $m=0.05$ and $m=0.2$ the only stable fixed point is $s=1$ (complete collapse). For $m=0.4$ there are two stable fixed points, but the initial fraction of defaults is almost zero, so the dynamics can only reach the left stable fixed point. (Right) The fraction $s$ of defaults is plotted in gray scale as a function of diversification degree $k$ and average robustness $m$. In the gray area cascades are large (over $90 \%$ of defaults). In the white area cascades are small (below $5 \%$ of defaults). The top left inset refers to the initial distribution with $m=0.2$ (see curve in left panel): no cascade occurs. The bottom right inset refers to the initial distribution with $m=0.4$ : a full cascade occurs.

where

$$
F\left(s, m, \sigma_{\eta}\right)=\sum_{j=1}^{k}\left(\begin{array}{l}
k \\
j
\end{array}\right) s^{j}(1-s)^{k-j} \Phi_{m, \sigma_{\eta}}\left(j \frac{a}{k}\right)
$$

\section{A stable fixed point always exists.}

The proof rests on the assumption that the defaults of the neighbors of a given agent are statistically independent, so that we can describe the probability of multiple defaults among the neighbors of an agent with a binomial distribution. This assumption is strictly valid only when there are few defaults in the system. However, when there are instead many defaults, the result shows that eventually the cascade involves the whole network. Thus, the presence of correlation becomes irrelevant and the result is still correct.

As an example, Fig. 2 (left) shows a plot of the R.H.S. of Eq. (14) as a function of the cascade size, for some specific parameters. Notice that with average robustness $m=0.4$ there are two stable fixed points and the lower one is reached, corresponding to no cascade. After decreasing $m$ to 0.2 , there is only one stable fixed point at $s=1$, corresponding to full cascade. Proposition 4 implies that there are only two regimes, one with only small cascades and one with only large cascades. Indeed, in Fig. 2 (right) the size of the cascade is plotted as a function of both $k$ and $m$. In the gray area cascades are large (over $90 \%$ of defaults). In the white area cascades are small (below 5\% of defaults). The two insets refer to two of the cases illustrated in Fig. 2 (left). If the initial distribution of robustness has a mean $m$ close to 0 and a large variance (small $k$ ), then obviously many firms have defaulted already at the beginning, when the cascade process starts (see lower inset of the figure). They affect other firms, many of which have also small robustness since the mean of the distribution is low, thus the resulting cascade is large.

Notice that, for any fixed $m$, increasing diversification is beneficial in the sense that at some point the cascade size drops to 0 . This is because as $k$ increases, the width of the distribution shrinks so that the fraction of agents initially defaulting becomes very small and very few or none of the other agents is weak enough to be dragged into a cascade. As a comparison, Gai and Kapadia (2010) find that contagion occurs in a window of levels of risk diversification, but for diversification large enough, both the frequency and the extent of the contagion go to zero.

\subsection{Interplay of robustness evolution and cascades. Implications on systemic risk}

Since systemic risk is the probability that a large fraction of agents in the financial system fail at the same time, it is now crucial to understand how frequently the system is found in the conditions that lead to a large cascade. Hence, we aim at estimating the probability $P^{c}$ of occurrence of large cascades, i.e. cascades involving at least a given fraction $s_{c} \in[0,1]$ of the system. In doing so, we want to take into account that robustness is not a static quantity and that the distribution of robustness that is likely to be realized at the beginning of a cascade evolves in time.

When banks default on their obligations and trigger a cascade, this occurs at a time scale (e.g., days) which is much faster than the evolution of robustness described by the dynamics introduced in Section 2. We thus assume a repeated two-stage process in which robustness evolves according to the diffusion dynamics of Eq. (11) until a default occurs. At this point the cascade dynamics of Eq. (13) starts, taking as initial condition the current distribution of robustness that resulted from the diffusion.

Systemic risk can be thus seen as the result of two factors: the size of a cascade which depends on the initial distribution of robustness, and the frequency by which the system visits the distribution of robustness that leads to such a 
cascade. The phase diagram of Fig. 2 (right) shows that a fraction of simultaneous defaults $s$ close to 1 occurs whenever the average robustness $m$ is below the boundary of the region of large cascades (in gray). Recall that the values of $\sigma$ and $k$ are constant during the evolution of $\eta_{i}(t)$. Thus, the expected time it takes during the evolution for the average robustness to reach the value $m$ can be approximated by the first passage time at $\eta=m$ of a representative trajectory of robustness starting from $\eta=1$. This approximation makes sense because of the mean reversion which keeps trajectories close to each other, as discussed earlier. Therefore, in this approach, the probability $P_{f}^{(c)}$ of cascades larger than $s_{c}$ is obtained as the inverse of the first passage time at a value $m$ such that a cascade larger than $s_{c}$ is triggered. The probability of large cascades estimated in this way turns out to be a non-monotonic function of the risk diversification. In other words, similar to the individual default probability, even the systemic default probability displays an intermediate optimal degree of diversification beyond which a further diversification becomes detrimental. This is stated in the following proposition.

Proposition 5. Consider the cascade process of Eq. (13). Assume the initial distribution of robustness is Gaussian $p(\eta, \tau=0)=\operatorname{Gauss}(m, \sigma)$. Assume the network of firms is a regular random graph with degree $k$. In mean-field approximation, the probability $P^{c}$ of occurrence of a systemic default involving a fraction of the system larger than $s_{c} \in[0,1]$, is

$$
P^{c}(k)=\frac{1}{T^{c}(k)}
$$

where

$$
\begin{aligned}
& T^{c}(k)=\min _{m}\left\{T(k, m) \mid S(k, m) \geq s_{c}\right\} \\
& T(k, m)=\frac{1-m}{\alpha q}+\frac{\sigma^{2}}{2 \alpha^{2}} \frac{\left(\exp \left(-2 \alpha k q(1-m) / \sigma^{2}\right)-1\right)}{k q^{2}}
\end{aligned}
$$

$S(k, m)$ is the cascade size solution of Eq. (14)

As a function of $k, P^{c}$ is not, in general, decreasing monotonically, and it has a unique minimum for several parameter settings.

As shown by the plot in Fig. 3, the probability of large cascades is not decreasing monotonically with $k$. On the contrary it exhibits a marked minimum for intermediate values of $k$. Notice that, as shown in Fig. 2, the cascade size $s$ has essentially two ranges of values: $s<0.05$ (i.e. $5 \%$ of agents fail) in the regime of small cascades and $s>0.9$ (i.e. $90 \%$ of agents fail) in the regime of large cascades. Therefore, the second point of the above proposition actually holds independently of the value $s_{c}$ taken as definition of "large cascade", as long as $s_{c}>0.05$.

Finally, the results of the paper can be summarized in the following conclusive corollary.

Corollary 1 (Non-monotonicity of systemic risk). In the presence of financial acceleration

1. The systemic risk is in general not monotonically decreasing with the risk diversification.

2. With larger diversification, the financial system is more likely to be near the threshold at which large cascades occur.

3. At least in some intervals of the range of the parameters involved, there exist an optimal level of systemic risk, which does not coincide with full diversification.

The statements above hold both in the absence and in the presence of external effects of defaults.
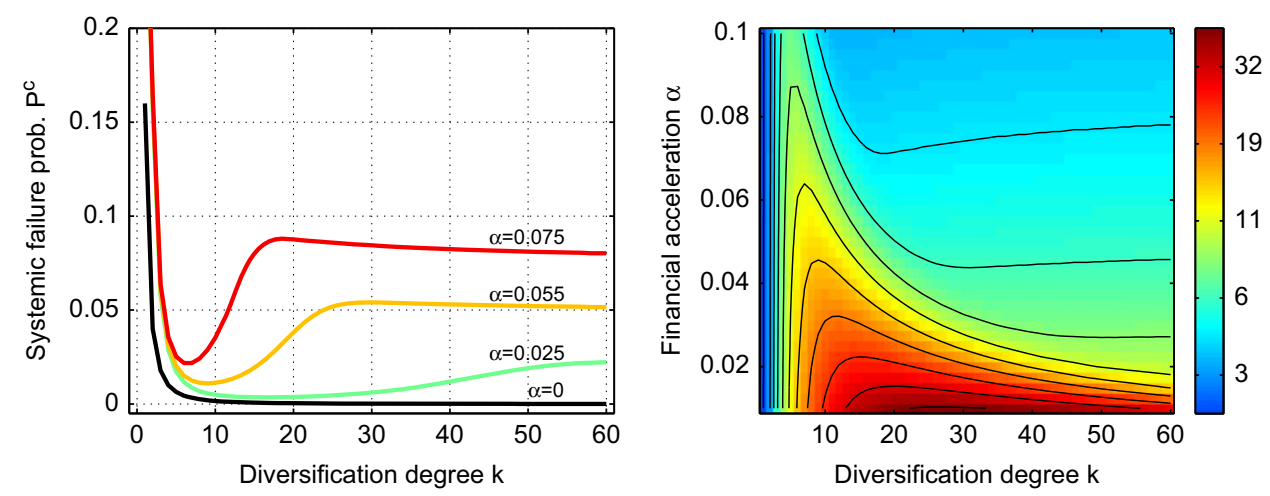

Fig. 3. (Left) Probability $P^{c}$ of occurrence of large cascades as a function of risk diversification $k$ for some values of the intensity $\alpha$ of financial acceleration. (Right) Expected time $T^{c}$ to large default cascades plotted in color code (log scale) as a function of $k$ and $\alpha$. The scale on the color bar indicates the range of the expected time to default in the time units of Eq. (11). Values of the parameters: $\epsilon=1$ and $\sigma=0.25$. $\alpha$ varies in steps of 0.0001 . The isoclines in black show that for any given $\alpha$, by increasing $k$ the expected time to default has an intermediate maximum. (For interpretation of the references to color in this figure caption, the reader is referred to the web version of this article.) 


\section{Conclusions}

In this paper, we have characterized the evolution over time of a credit network in the most general terms as a system of coupled stochastic processes, each describing the dynamics of individual financial robustness. The coupling comes from the fact that agents assets are other agents' liabilities. Thus, each agent's financial robustness is inter-dependent with the financial robustness of the counterparties through risk sharing as well as external effects arising from defaults.

Our goal is to investigate possible reasons why diversification may not deliver the expected benefits. It is relatively easy to do so by assuming a disease-like contagion, or a cost that is supra-linear in the number of joint defaults. Here, instead, we have provided an explanation based on a milder assumption. We find that financial acceleration, i.e. the positive feedback of financial robustness on itself, is a sufficient condition. It eventually more than offsets the stabilizing role of risk sharing and amplifies the effects of a shock to a single agent of the network, leading to a full fledged systemic crisis. The relationship between the probability of default, both individual as well as systemic, and connectivity is U-shaped. If connectivity is already high, a further increase may have the perverse effect of amplifying financial distress through the financial acceleration and to increase systemic risk.

The present work aims to provide a basic framework and can be extended in several directions. First of all one could investigate other variants of the mechanisms of both distress propagation and financial acceleration to possibly extend the validity of these results. Second, one could study the effect of different network architectures. In particular, skewed degree distribution are known to play a role in spreading phenomena, as shown in technological diffusion on social networks (Jackson and Rogers, 2007). Another open question in our context concerns the systemic risk of a network organized in clusters which are densely connected internally but loosely connected among each other. Whether there exists an optimal level of clusterization would have important implications on the debate about the role and design of globalization (Stiglitz, forthcoming). Finally, one could model the set of contracts among agents as an endogenously evolving network (Jackson, 2008; Goyal, 2007; Castiglionesi and Navarro, 2010; Allen et al., 2011) in which each agent decide strategically which ties to form or to severe, based on the expected utility they provide.

\section{Appendix A. Proofs of Propositions}

Proof of Proposition 1. We derive a recursive expression of the probability $q_{t}$ that the financial acceleration takes place in a given discrete time $t \in \mathcal{N}$, that is, $q_{t}=P\left\{h_{i}(t)=-\alpha\right\}$. For the variation in robustness over the interval [t-1,t], by neglecting the mean reversion term $\bar{\eta}_{i}-\eta_{i}$ in Eq. (11), we have

$$
\begin{aligned}
& \eta_{i}(t-d t)-\eta_{i}(t-1-d t)=\int_{t-1-d t}^{t-d t} d \eta_{i}(s) d s \\
& \sigma \int_{t-1-d t}^{t-d t} d \xi_{i}+\int_{t-1-d t}^{t-d t} h(t) \delta(t-s) d s=\sigma \zeta \sqrt{1}+h(t-1)
\end{aligned}
$$

where $\sigma \zeta \sqrt{1}$ represents the accumulated Wiener shock over the interval $[t-1, t]$. Then,

$$
\begin{aligned}
& q_{t}=P\left\{h_{i}(t)=-\alpha\right\}=P\left\{\eta_{i}(t-d t)-\eta_{i}(t-1-d t)<-\epsilon \sigma\right\} \\
& q_{t}=P\{\sigma \zeta+h(t-1)\}=P\left\{\zeta<-\frac{h(t-1)}{\sigma}-\epsilon\right\}
\end{aligned}
$$

There are two possible cases now. In the first case, it is $h(t-1)=-\alpha$, occurs with probability $q_{t-d t}$. In the second case, it is $h(t-1)=0$, which occurs with probability $1-q_{t-d t}$. In both cases, the probability that $\zeta$ is smaller than a certain value $x$ is given by the standard cumulative Gaussian distribution at $x, \Phi(x)$, because $\zeta$ is a Gaussian stochastic variable with zero mean and standard deviation 1 . Therefore,

$$
q_{t}=q_{t-d t} \Phi\left(\frac{\alpha}{\sigma}-\epsilon\right)+\left(1-q_{t-d t}\right) \Phi(-\epsilon)
$$

Solving for the fixed point $q$ one finds

$$
q=\frac{\Phi(-\epsilon)}{1-\Phi\left(\frac{\alpha}{\sigma}-\epsilon\right)+\Phi(-\epsilon)}
$$

where $\Phi$ is the cumulative distribution function of the Gaussian. To show that the fixed point is stable we proceed as follows. Denote the R.H.S. of Eq. (A.3) as $f(q)=q_{t-1} \Phi_{2}+\left(1-q_{t-1}\right) \Phi_{1}$. Notice that both cumulative distributions $\Phi_{1}$ and $\Phi_{2}$ lay in ]0,1[ for finite values of the parameters. Since $f$ is linear in $q$ and $f(0)>0$, a unique stable fixed point exists iff $d f / d q<1$. This is always verified because $d f / d q=\Phi_{2}-\Phi_{1}<1$.

Proof of Proposition 2. In the proof of Proposition 3 the first passage time is derived in the case with generic diversification level $k$. In the context of the present proposition take simply $k=1$ and probability $q$ as in Eq. (A.3). Both terms of the first passage time in Eq. (A.11) are decreasing with $\alpha$, hence the conclusion. 
Proof of Proposition 3. (1) The mean first passage time of a random walk with variance $\sigma_{r}^{2}$ is known to be $T_{f}=1 / \sigma_{r}^{2}$. We apply this result to our case with $\sigma_{r}=\sigma / \sqrt{k}$ and we obtain $P_{f}(k)=1 / T(k)=\sigma^{2} / k$.

(2) We recall the following result on SDE (see for instance, Gardiner, 2004, p. 139). The mean first passage time of an one-dimensional homogeneous SDE

$$
d x=A(x) d t+\sqrt{B(x)} d \xi
$$

with absorbing barrier at $x=a \geq 0$ and repulsive barrier at $x=b>a$ and initial value $x$ is

$$
T(x)=2 \int_{a}^{x} \frac{d y}{\psi(y)} \int_{y}^{b} \frac{\psi(z) d z}{B(z)}
$$

where

$$
\psi(x)=\exp \left(\int_{a}^{x} \frac{2 A\left(x^{\prime}\right) d x^{\prime}}{B\left(x^{\prime}\right)}\right)
$$

In our case, both the drift and diffusion terms are constant: $A(x)=A=-\alpha q$ and $B(x)=B=\sigma^{2} / k$. The computation of the integral proceeds as follows:

$$
\begin{aligned}
& \psi(x)=\exp \left(\frac{2 A(x-a)}{B}\right) \\
& \begin{aligned}
& \int_{y}^{b} \frac{\psi(z) d z}{B(z)}=\frac{1}{2 A}[\exp (2 A(b-a) / B)-\exp (2 A(y-a) / B)] \\
& 2 \int_{a}^{x} \frac{d y}{\psi(y)} \int_{y}^{b} \frac{\psi(z) d z}{B(z)}=2 \int_{a}^{x} \frac{1}{2 A} \exp (2 A(y-a) / B)^{-1}[\exp (2 A(b-a) / B)-\exp (2 A(y-a) / B)] \\
&=\frac{1}{A} \exp (2 A(b-a) / B) \int_{a}^{x} \exp (-2 A(y-a) / B)-\frac{1}{A} \int_{a}^{x} d y
\end{aligned}
\end{aligned}
$$

We finally obtain

$$
T(x)=\frac{B}{2 A^{2}} \exp (2 A(b-a) / B)[1-\exp (-2 A(x-a) / B)]-\frac{x-a}{A}
$$

In the context of our model it is $a=0, b=1$. Moreover, we assume $x=1$, which implies that firms are created with the highest robustness. This is a conservative hypothesis with respect to the result which we will obtain. Substituting for the values of $a, b, A, B$, we obtain

$$
T_{f}=\frac{1}{\alpha q}+\frac{\sigma^{2}}{2 \alpha^{2}} \frac{\left(\exp \left(-2 \alpha k q / \sigma^{2}\right)-1\right)}{k q^{2}}
$$

Bearing in mind that $q=q(k)$, the derivative of the mean first passage time with respect to the degree $k$ can now be computed as follows. We denote the derivative of a function $f$ equivalently as $D_{k f}$ or $f^{\prime}$

$$
\begin{aligned}
T_{f}^{\prime} & =\frac{-q^{\prime}}{\alpha q^{2}}+\frac{\sigma^{2}}{2 \alpha^{2} k^{2} q^{4}}\left[\exp \left(\frac{-2 \alpha k q}{\sigma^{2}}\right)\left(\frac{-2 \alpha}{\sigma^{2}}\right) D_{k}[k q] k q^{2}-\left(\exp \left(\frac{-2 \alpha k q}{\sigma^{2}}\right)-1\right) D_{k}\left[k q^{2}\right]\right] \\
& =\frac{-q^{\prime}}{\alpha q^{2}}+\frac{\sigma^{2}}{2 \alpha^{2} k^{2} q^{4}}\left[\exp \left(\frac{-2 \alpha k q}{\sigma^{2}}\right)\left(\frac{-2 \alpha}{\sigma^{2}}\right)\left(q+k q^{\prime}\right) k q^{2}-\left(\exp \left(\frac{-2 \alpha k q}{\sigma^{2}}\right)-1\right)\left(q^{2}+2 k q q^{\prime}\right]\right. \\
& =\frac{-q^{\prime}}{\alpha q^{2}}+\frac{\sigma^{2}}{2 \alpha^{2} k^{2} q^{4}}\left(f_{0}\left(\frac{-2 \alpha}{\sigma^{2}}\left(q+k q^{\prime}\right) k q^{2}-\left(q^{2}+2 k q q^{\prime}\right)\right)+\left(q^{2}+2 k q q^{\prime}\right)\right) \\
& =\frac{-q^{\prime}}{\alpha q^{2}}+\frac{\sigma^{2}}{2 \alpha^{2} k^{2} q^{4}}\left(-f_{0}\left(f_{1}+f_{2}\right)+f_{2}\right)
\end{aligned}
$$

where

$$
\begin{aligned}
& \Phi_{1}=\Phi(-\epsilon), \quad \Phi_{2}=\Phi\left(\frac{\alpha \sqrt{k}}{\sigma}-\epsilon\right) \\
& \Phi(x)=\frac{1}{\sqrt{2 \pi}} \int_{-\infty}^{x} \exp \left(-t^{2} / 2\right) d t, \quad \Phi_{2}^{\prime}=\frac{\alpha k^{-1 / 2}}{2 \sigma \sqrt{2 \pi}} \exp \left(-\frac{(\alpha \sqrt{k} / \sigma-\epsilon)^{2}}{2}\right) \\
& q=\frac{\Phi_{1}}{1-\Phi_{2}+\Phi_{1}}, \quad q^{\prime}=\frac{\Phi_{1} \Phi_{2}^{\prime}}{\left(1-\Phi_{2}+\Phi 1\right)^{2}}
\end{aligned}
$$




$$
f_{0}=\exp \left(\frac{-2 \alpha k q}{\sigma^{2}}\right), \quad f_{1}=\frac{2 \alpha}{\sigma^{2}}\left(q+k q^{\prime}\right) k q^{2}, \quad f_{2}=q^{2}+2 k q q^{\prime}
$$

In order to determine in general the existence and the value of the maxima of $T(k)$, we solve the equation $T(k)=0$, with $T(k)^{\prime \prime}<0$. Since the expression contains the error function, the solution of the equation is necessarily numerical. We fix the parameter $\epsilon=\{1,2\}$ and we vary $(\sigma, \alpha)$ in $[0.05,1] \otimes[0.05,1]]$. The results shown here refer to a step size of 0.001 . However, the results were unaffected by using even finer resolutions. For each pair $(\sigma, \alpha)$ we determine the values of $k$ where $T^{\prime}(k)$ changes from positive to negative sign. The idea is illustrated in Fig. A1 (left) for a fixed parameter set, as an example. The functional dependency of $T$ and $T^{\prime}$ on $k$ displays a non-monotonic behavior. In particular, in the inset, $T^{\prime}$ crosses the value of zero from above in the point of maximum (indicated by an arrow). The general result is illustrated in Fig. A2 (left). For each pair $(\sigma, \alpha)$ in the explored grid we always find a unique maximum. However, the point of maximum has to be larger than 1 , to be a meaningful level of diversification. The gray area indicates the region where the maximum of $T$ occurs at $k=1$. In the white area it occurs at $\infty>k>1$. As it can be seen, $\alpha \leq \sigma$ is a sufficient condition to have existence and uniqueness of a maximum at $k>1$. Fig. A2 (right) shows the value of the optimal degree as a function of $\sigma, \alpha$. This is typically smaller than 30 but it grows fast to larger values if the intensity $\alpha$ of the financial acceleration is very small.

The implication of the result are further illustrated in Fig. A1 (right). The value of $\sigma$ is fixed and the dependence of $T_{f}$ on $k$ shows a point of maximum for each value of $\alpha$, as indicated by the isoclines. The existence of a maximum for $T_{f}$ implies a minimum of the probability $P_{f}$ of default. The procedure employed samples the parameter space at discrete values and thus does not provide a proof that for any value in the explored range there exists a maximum for $T_{f}$. The analysis shows, by existence, that there are very many parameter values where this is the case. Because $T_{f}$ is continuous in $\alpha$, with continuous derivatives for $\alpha>0$, one could conjecture the existence of a maximum of $T_{f}$ at least in the range of most interest to the model, i.e. $\alpha \in] 00.15$. These are realistic values of acceleration which already produce a significant effect on $T_{f}$.
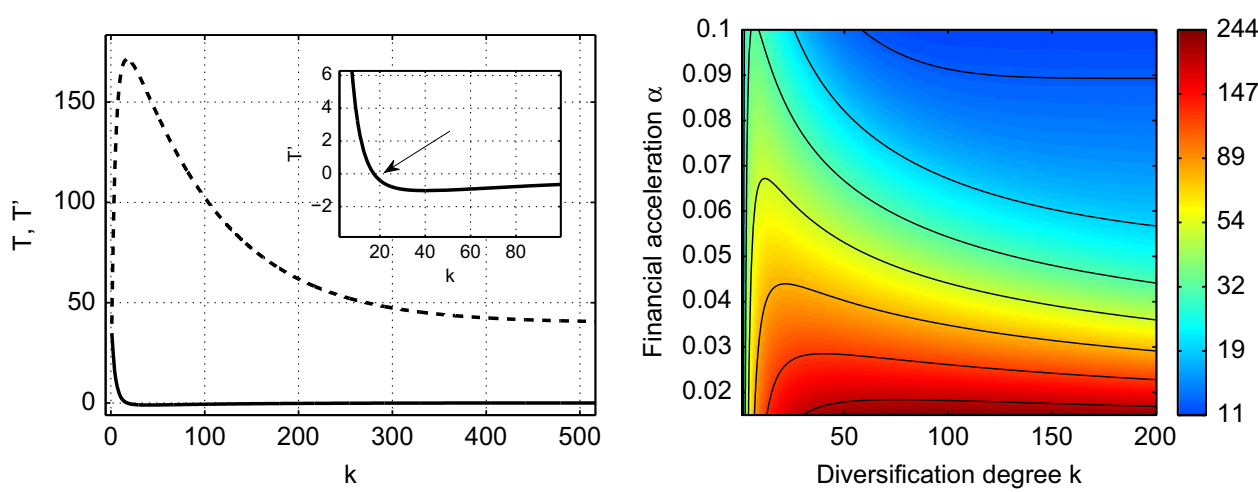

Fig. A1. (Left) Dependency on the degree $k$ of the mean first passage time $T(k)$ (solid line) and its derivative $T(k)^{\prime}($ dashed line). Parameter values: $\alpha=0.025, \epsilon=1, \sigma=0.15$. (Right) Expected time to default plotted in color code (log scale) as a function of $k$ and $\alpha$. The scale on the color bar indicates the range of the expected time to default in the time units of Eq. (11). Values of the parameters: $\epsilon=1$ and $\sigma=0.25$. $\alpha$ varies in steps of 0.0001 . The isoclines in black show that for any given $\alpha$, by increasing $k$ the expected time to default has an intermediate maximum. (For interpretation of the references to color in this figure caption, the reader is referred to the web version of this article.)
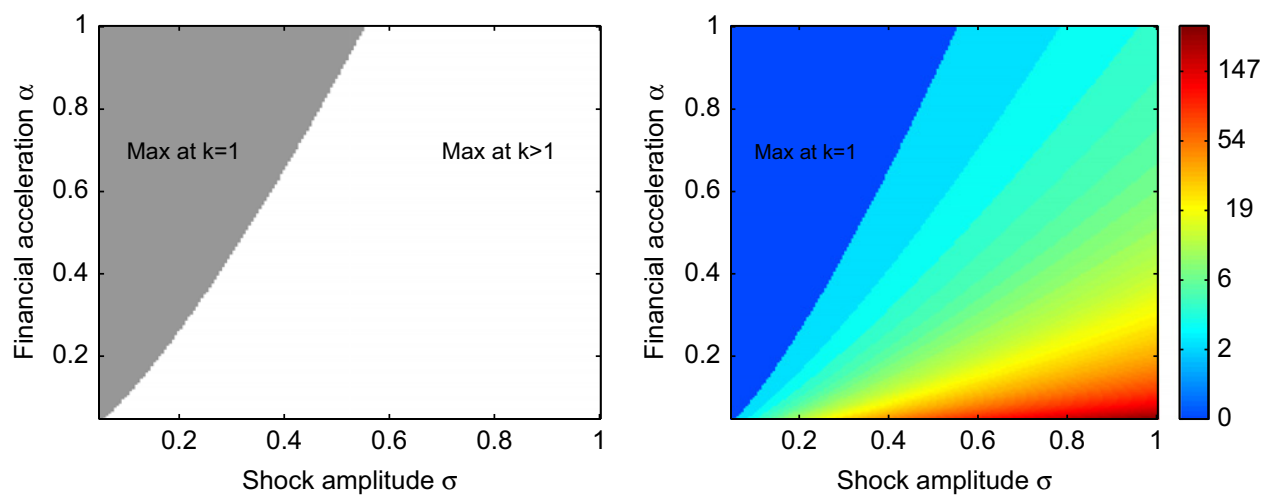

Fig. A2. Left: Number of maxima of $T(k)$. For each pair of values $\sigma, \alpha$ the color code illustrates the number of values of $k$ where the derivative $T^{\prime}(k)$ changes from positive to negative values. In the inset, $T^{\prime}$ crosses the value of zero from above in the point of maximum (indicated by an arrow). Right: Value of $k$ in the point of maxima of $T(k)$ for each pair of values of the parameters $\sigma, \alpha$. (For interpretation of the references to color in this figure caption, the reader is referred to the web version of this article.) 
Proof of Proposition 4. The specific cascading process in Eq. (13) is modeled as follows:

$$
\eta_{i}(\tau+1)=\eta_{i}(\tau)-\frac{a}{k} \sum_{j=1}^{k} \delta_{j}(\tau)
$$

where $\delta_{j}(\tau)=1$ iff $j$ fails at time $\tau$. An equivalent but more convenient expression is

$$
\eta_{i}(\tau+1)=\eta_{i}(0)-\frac{a}{k} \sum_{j=1}^{k} \delta_{j}^{c}(\tau)
$$

where $\delta_{j}^{c}(\tau)=1$ iff $j$ has failed at any time in $[0, \tau]$. We ask what is the cumulative fraction of agents that have failed so far at time $\tau$. This is

$$
n(\tau+1)=\operatorname{Pr}\left\{\eta_{i}(\tau+1)<\theta\right\}=\operatorname{Pr}\left\{\sum_{j=1}^{k} \delta_{j}^{c}(\tau)>\eta_{i}(0)\right\}
$$

For simplicity, we assume that the network is a regular graph with degree $k$. Whether a given agent $i$ fails by the time step $\tau+1$ depends on the number $k_{f}$ of the neighbors that have already failed, out of the total number $k$ of neighbors. At time step $\tau+1$, the possible events are $k_{f}=\sum_{j=1}^{k} \delta_{j}^{c}(\tau)=1,2, \ldots, k$. In each of these events, the probability that agent $i$ fails depends on the initial value of its robustness. Assume all defaults that have occurred so far are uncorrelated across agents. Then, they follow a binomial distribution, $\operatorname{Pr}\left\{k_{f}\right.$ defaults among $k$ neighbors $\}=\left(\begin{array}{l}k \\ k_{f}\end{array}\right) p^{k_{f}}(1-p)^{k-k_{f}}$, where $p$ is the probability that any given agent has failed so far. In the limit of a large network it is $p=n(\tau)$. Finally, we need to take into account that firms do not recover during the cascade and thus the fraction of default can only increase. Therefore,

$$
n(\tau+1)=\max \left\{s_{0}, \sum_{k_{f}=1}^{k}\left(\begin{array}{c}
k \\
k_{f}
\end{array}\right) n(\tau)^{k_{f}}(1-n(\tau))^{k-k_{f}} \operatorname{Pr}\left\{\eta_{i}(\tau) \leq \frac{a k_{f}}{k}\right\}\right\}
$$

where $s_{0}$ is the initial fraction of defaults. This is a recursive equation of the type $n(\tau+1)=F(n(\tau))$. Once the probability distribution of $\eta$ is specified, the fixed points are the solutions of $n=F(n)$. For example, in the case of regular graph, with $\eta$ following a Gaussian distribution with mean $\mu$ and standard deviation $\sigma$ we obtain

$$
\begin{aligned}
& n(\tau+1)=\max \left\{s_{0}, \sum_{k_{f}=1}^{k}\left(\begin{array}{c}
k \\
k_{f}
\end{array}\right) n(\tau)^{j}(1-n(\tau))^{k-k_{f}} \frac{1}{\sigma \sqrt{2 \pi}} \int_{-\infty}^{a k_{f} / k} \exp \left(-\frac{(u-\mu)^{2}}{2 \sigma^{2}}\right)\right\} \\
& n(\tau+1)=\max \left\{s_{0}, \sum_{k_{f}=1}^{k}\left(\begin{array}{c}
k \\
k_{f}
\end{array}\right) n(\tau)^{k_{f}}(1-n(\tau))^{k-k_{f}} \frac{1}{2}\left(1+\operatorname{erf}\left(\frac{a k_{f} / k-\mu}{\sigma \sqrt{2}}\right)\right)\right\}
\end{aligned}
$$

Notice that $F(n) \geq s_{0}$ with $s_{0}>0$ strictly for $\mu<\infty$ and $\sigma>0$. Since in addition $F(n)$ is non-decreasing, there exists at least one stable fixed point. There maybe more than one but what matters here is only the smallest stable fixed point $s$ with $s \geq s_{0}$. The equation above can be solved numerically with arbitrary precision for any choice of the parameters. The results are shown in Fig. 2.

In general it would also be possible to account for heterogeneous degree distribution. This requires, however, a more extended analytical treatment that goes beyond the objective of this paper.

Proof of Proposition 5. Let us fix the amplitude $\sigma$ of the shocks. The probability $P^{c}$ that a fraction $c$ of agents fail in the same period, possibly due to a cascade, can be computed as follows. We use the same general result on mean first passage time described in proof of Proposition 4, Eq. (A.8). In the present context, we are interested in the first passage time at the value $\eta=m$ starting from the upper barrier $\eta=b=1$

$$
T(k, m)=2 \int_{m}^{b} \frac{d y}{\psi(y)} \int_{y}^{b} \frac{\psi(z) d z}{B(z)}
$$

Substituting for $a=m$ and $b=1$ in Eq. (A.10) we obtain

$$
T_{f}=\frac{1-m}{\alpha q}+\frac{\sigma^{2}}{2 \alpha^{2}} \frac{\left(\exp \left(-2 \alpha k q(1-m) / \sigma^{2}\right)-1\right)}{k q^{2}}
$$

The probability that over time the robustness following Eq. (11) reaches the value $m$ is given by the $1 / T(k, m)$. In a mean field approach we take this as the probability that the average robustness $\bar{\eta}$ in the system reaches the value $m$.

On the other hand, the solution $S(k, m)$ of Eq. (14) gives the expected size of the cascade occurring when the average robustness in the system reaches the value $m$. We are interested now in the mean first passage time $T^{c}(k)$ to reach the point where the occurring cascade is larger than $c$

$$
T^{c}(k)=\min _{m}\{T(k, m) \mid S(k, m) \geq c\}
$$


Consequently, $P^{c}(k)=1 / T^{c}(k)$ is the probability of occurrence of a cascade larger than $c$, which is a possible measure of systemic risk. The function of $T^{c}(k)$ in Eq. (A.25) cannot be expressed in closed form. However, it can be computed numerically with arbitrary precision for any choice of the parameters $\alpha, \sigma, \epsilon$. As an example, Fig. 2 (left) shows the dependence of $T^{c}$ on $k$ for given parameter values. The curve displays a maximum, which implies a minimum for the probability $P^{c}(k)$.

Differently from what done in the proof of Proposition 3, here there is no closed-form expression for the derivative of $T^{c}(k)$, and we thus compute numerically the point of maximum directly from the values of the function $T^{c}(k)$. We do so for all values of $\alpha$ varying in the interval [0.001,0.1] with steps of width 0.001 , and for fixed values $\epsilon=1,2, \sigma=0.4$ and $s_{c}=0.25$. Notice that, as already mentioned in Section 3.2, the result is very robust with respect to $s_{c}$ in virtue of the two regimes of cascades. As an illustration of the procedure, in Fig. 3 (right) the values of $T^{c}$ are plotted as a function of $k$ and $\alpha$ in the range of interest. It is apparent that for all values of $\alpha$ sampled in the interval there is one global maximum of the expected time to large cascades $T^{c}$ as a function of the degree $k$ of risk diversification. In other words, there is a value of optimal connectivity degree as a function of $\alpha$, which grows for decreasing values of $\alpha$.

As before, this procedure does not allow to claim that there is a maximum for any possible value in the sampled interval. It proves by existence that there are very many parameter settings where this is the case. Given the fine resolution of our sampling, this is a quite reasonable conjecture.

\section{Appendix B. Numerical simulations}

Let us refer to Eq. (1) as Model 1, or jump-diffusion model, and to Eq. (5) as Model 2, or drift-diffusion model. In this section we show by Monte Carlo computer simulations that the expected first passage time in Model 2 is a good approximation of the expected first passage time (MFPT) in Model 1.

Following the simple Euler discretization scheme, we compute the variation of the variable $\eta$, over steps $s$, each of size $\Delta$. Jumps occur, possibly, only every $n$ steps, with $n \in \mathcal{N}$ and with $\Delta n=1$. We can then reduce the step size by increasing $n$. With a slight abuse of notation we call $h$ the function that overall represents the jump. Then,

$$
\eta(s+\Delta)-\eta(s)=\frac{\sigma}{\sqrt{k}} \xi \sqrt{\Delta}+h(s)
$$

with

$$
h(t)= \begin{cases}-\alpha & \text { if } \left.\bmod (s, n)=0 \text { and } \eta_{(} s-\Delta\right)-\eta(s-n-\Delta)<-\epsilon \frac{\sigma}{\sqrt{k}}, \\ 0 & \text { otherwise }\end{cases}
$$

where $\bmod (s, n)$ is the remainder of the division of $s$ by $n$. Notice that when $\bmod (s, n)=0$, then $s-\Delta$ is right before the step in which the conditional shock in $s$ can possibly occur, while $s-n-\Delta$ is right before the conditional shock in $s-n$. Thus the difference is affected by the previous conditional shock at $s-n$, but not yet by the conditional shock that will occur in $s$. The scheme above is illustrative of the method but it is not meant to be a pseudo-code of the computer program. We also implement a reflective barrier at 1 and an absorbing barrier at 0 , as in the model.

We run Monte Carlo simulations of sample paths of the process, starting from 1 , and we measure the time step $T$ at which $\eta$ becomes 0 or negative. At this point we start a new sample path. We repeat this procedure a number $N_{\text {runs }}$ of times and we take the average time across runs. We make sure that the simulation time is long enough that in all runs the process eventually exit from the lower barrier. We then compute the mean $T$ across all the runs.

We compare the theoretical value of the drift-model with the empirical value of the simulated jump-diffusion model. Fig. B1 reports the results of MFPT with $\Delta=0.0001$ and $N_{\text {runs }}=1000$. The theoretical value in red are computed from Eq. (A.11), with the expression for $q(k)$ in Eq. (A.6), as obtained in Proposition 1. The empirical value is in blue. The dotted blue curves represent the average $T$ plus(minus) the standard deviation of $T$ across runs. Notice the large standard deviation reflecting a great variability from run to run in the region of intermediate $k$. And yet notice the good agreement of the mean (blue) with the theory. This picture shows that in the intermediate $k$ regime because the trend reinforcement is weak, $q \ll 1$ and the average drift is small $\alpha q \ll \alpha=0.01$. Thus, both the red and the blue curves are close to the case with virtually no drift (upper green curve). In contrast, with large $k, q \rightarrow 1$ and the average drift is close to value of the constantdrift $\alpha$ (lower green curve).

The systematic study of MFPT for Model 1 poses some computational challenges if one wants to explore the space of parameters with fine granularity. Thus Model 2 offers a good tool to do this via an analytical approximation.

\section{Appendix C. A simple calibration exercise}

An accurate calibration of the model is beyond the scope of this paper. However it is worthwhile to illustrate how a simple exercise can be carried out and what kind of conclusions can be drawn.

The first challenge in the calibration of our model is the scarcity of data on exposures among financial institutions, which is highly confidential information. In particular, exposures among global institutions are not known, although they could be considered as the most relevant for systemic risk. It would of course be very interesting to know how the average 


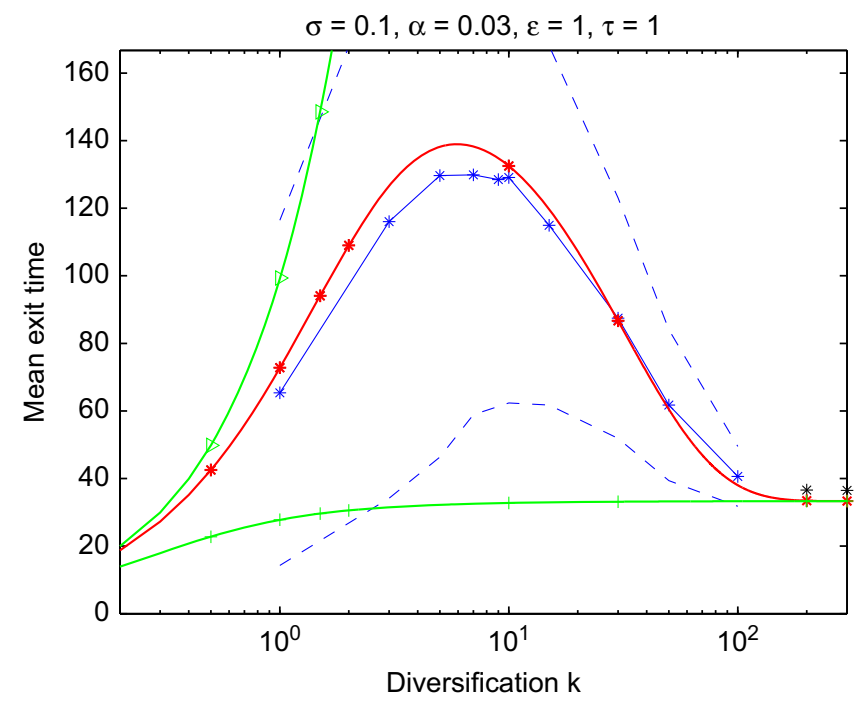

Fig. B1. Mean first passage time $T$ computed with $\Delta=0.0001$ and $N_{\text {runs }}=1000$. For all curves, $\sigma=0.1$. Red curve: Model 2 (drift-model), with $\alpha=0.03$. Blue curve: simulation of Model 1 (jump-diffusion model) with same $\alpha$. Blue dotted curves: $T \pm \sigma_{T}$. Green upper curve: theoretical value with constant drift $a=0.0001$. Green lower curve: theoretical value with constant drift $a=0.03$. (For interpretation of the references to color in this figure caption, the reader is referred to the web version of this article.)

connectivity has evolved in the last 10 years and since the unraveling of the financial crisis. However, it is simply not possible to measure accurately the number of relevant connections in the financial system.

For instance, some empirical studies of financial contagion on national credit networks (Degryse and Nguyen, 2007; Mistrulli, 2011; Upper and Worms, 2004) do not report information on the connectivity of the network. It should be emphasized that our model is well suited to be applied to the core of a core-periphery structure because the density of connections in the core justifies the mean-field approach taken in this paper. Empirical work on the FED payment system (Soramaki et al., 2007) suggests that in the US there is a core-periphery structure. The core comprises 25 among the largest institutions which are densely connected among each other, with average degree $11 \pm 8$. Similar insight can be obtained from the network diagrams of Cont et al. (2010), although they do not focus on the core-periphery structure. They report an average in-degree and out-degree around 8, which increases if one would select the top institutions.

On the other hand, the only available data on potential interdependencies among global players concerns ownership relations. Recent empirical work has shown that at the global level there is a very marked core-periphery structure with a tightly knit core (Vitali et al., 2011) comprising the largest global financial institutions. Within this set, we have further selected the global institutions that received financial aid through the FED emergency programs in 2008-2009 (Battiston et al., under revision). We obtained a group of 36 institutions with an average degree $17 \pm 13$.

A second challenge is finding an empirical financial ratio that (1) fits the type of dynamics of Eqs. (11) and (2) for which time series of suitable length and frequency are available. Moreover, the estimation of the parameters of the dynamics of our model from such time series is a non-trivial econometric analysis which deserves further work. Here, as an illustrative exercise, we examined the time series of equity (meant as market capitalization) of the set of 36 institutions selected as described earlier in a period of 1006 trading days from August of 2007 to July of 2009. After normalizing the equity of each institution to its own maximum value in time span of the data, each time series varies in the range $(011]$. As shown in Fig. C1, the time series display an evident negative trend, until they reach a minimum in March 2009 (around trading day 600 ). It is also evident that the strong correlation across the time series, which not only could come from an exogenous factors but could also come from the interdependence of the time series. We selected the period August 2007-March 2009 (trading days 1-600) - the decreasing phase - as representative of a situation of financial acceleration.

From the time series of each institution, we measured the drift and we took its average value 0.025 across institutions as an estimate of the parameter $\alpha$ in Eq. (11). From each time series, we measured also the standard deviation of the returns. We found an average value of 0.02 , which we have taken as an estimate of the parameter $\sigma_{z}$ of Eq. (11). In the model, this would be the volatility, net of the effect of the diversification. In order to estimate the underlying volatility $\sigma=\sqrt{k} \sigma_{z}$, we would need the average connectivity. This can be estimated only roughly from the empirical evidences mentioned above. Notice that the measured volatility could include other additional factors and it is probably overestimated (see below).

Fig. C1 (right) shows the mean expected passage time as a function of the degree for three different scenarios. In each scenario, we assume an average degree $k$ and we determine the optimal degree $k^{*}$. For instance, with degree $k=9$, we have $\sigma=0.02 \times \sqrt{9}=0.06$. The optimal average degree is $k^{*}=3.8$. Similarly, for $k=25$ we obtain $\sigma=0.1$ and $k^{*}=10.6$; for $k=56.2$ we obtain $\sigma=0.15$ and $k^{*}=23.9$. The fact that the volatility is probably overestimated, only accentuate this result, 

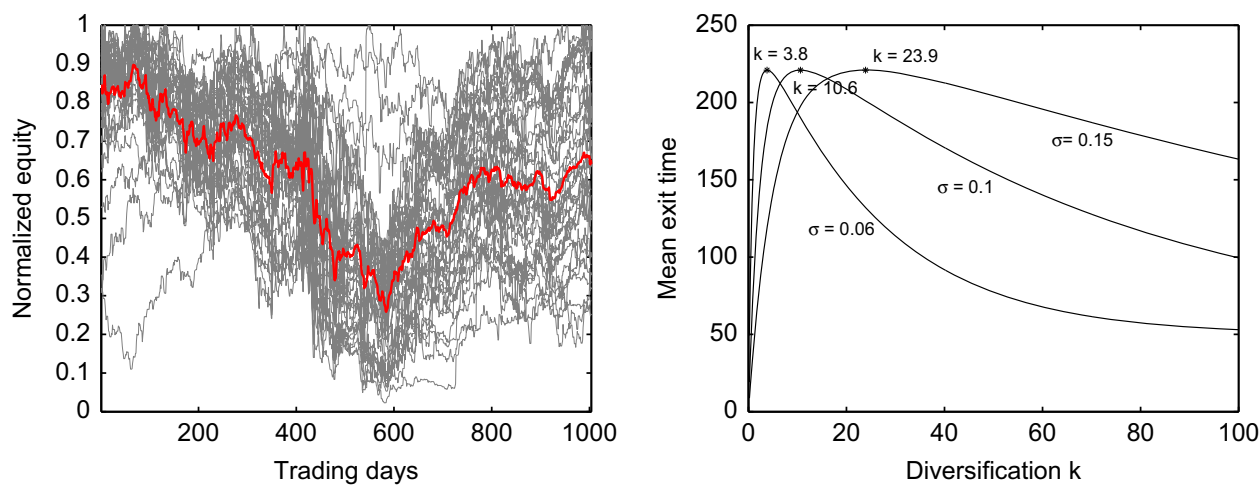

Fig. C1. Left: Normalized values of equity across 39 global financial institutions benefiting from the FED emergency programs in 2008. Right: Theoretical mean first passage time $T$ calibrated on the estimated average values of $\sigma$ and $\alpha$ for the network of such financial institutions. (For interpretation of the references to color in this figure caption, the reader is referred to the web version of this article.)

because the optimal degree would shift to the left. In other words, the results of the exercise suggest that, given the observed level of financial acceleration and volatility, the system is slightly over diversified with respect to the optimal level.

To conclude, we have presented a sketch of how one can carry out a calibration of the model. The results presented here should be taken only as an illustration what kind of implications could be drawn. A more systematic and careful estimation of the parameter is needed in order to provide more robust indications.

\section{References}

Allen, F., Babus, A., 2009. Networks in finance. In: The Network Challenge: Strategy, Profit, and Risk in an Interlinked World. Wharton School Pub., p. 367. Allen, F., Babus, A., Carletti, E., 2011. Asset commonality, debt maturity and systemic risk. Journal of Financial Economics. URL 〈http://www. sciencedirect.com/science/article/pii/S0304405X11001644>.

Allen, F., Gale, D., 2001. Financial contagion. Journal of Political Economy 108 (1), 1-33.

Bartram, S.M., Brown, G.W., Hund, J.E., 2007. Estimating systemic risk in the international financial system. Journal of Financial Economics 86 (3), 835-869. Battiston, S., Gatti, D.D., Gallegati, M., Greenwald, B.C.N., Stiglitz, J.E., 2007. Credit chains and bankruptcies avalanches in production networks. Journal of Economic Dynamics and Control 31 (6), 2061-2084.

Battiston, S., Puliga, M., Kaushik, R., Tasca, P., Caldarelli, G. DebtRank: too central to fail? Financial networks, the FED and systemic risk, Scientific Reports, in preparation.

Bernanke, B., Gertler, M., Gilchrist, S., 1999. The financial accelerator in a quantitative business cycle framework. In: Taylor, J., Woodford, M. (Eds.), Handbook of Macroeconomics. Amsterdam, North-Holland.

Black, F., Cox, J., 1976. Valuing corporate securities: some effects of bond indenture provisions. Journal of Finance 31 (2), $351-367$.

Boissay, F., 2006. Credit Chains and the Propagation of Financial Distress. Working Paper 573. European Central Bank.

Boss, M., Summer, M., Thurner, S., 2004. Contagion flow through banking networks. Lecture Notes in Computer Science, vol. 3038/2004; 2004, pp. 1070-1077.

Brunnermeier, M., 2008. Deciphering the 2007-08 liquidity and credit crunch. Journal of Economic Perspectives 23 (1), 77-100.

Brunnermeier, M., Pederson, L., 2009. Market liquidity and funding liquidity. Review of Financial Studies 22, 2201-2238.

Castiglionesi, F., Navarro, N., 2010. Optimal Fragile Financial Networks. SSRN Working Paper Series.

Cont, R., Moussa, A., Santos, E.B., 2010. Network Structure and Systemic Risk in Banking Systems.

Degryse, H., Nguyen, G., 2007. Interbank exposures: an empirical examination of contagion risk in the Belgian banking system. International Journal of Central Banking 3 (2), 123-171.

Diamond, D., Dybvig, P., 1983. Bank runs, deposit insurance, and liquidity. The Journal of Political Economy 91 (3), 401.

Eisenberg, L., Noe, T., 2001. Systemic risk in financial systems. Management Science 47 (2), 236-249.

Elsinger, H., Lehar, A., Summer, M., 2006. Risk assessment for banking systems. Management Science 52 (9), $1301-1314$.

Freixas, X., Parigi, B.M., Rochet, J.-C., 2000. Systemic risk, interbank relations, and liquidity provision by the central bank. Journal of Money, Credit \& Banking 32, 611-638.

Furfine, C., 2003. Interbank exposures: quantifying the risk of contagion. Journal of Money, Credit \& Banking 35 (1), $111-129$.

Gai, P., Kapadia, S., 2010. Contagion in financial networks. Proceedings of the Royal Society A: Mathematical, Physical and Engineering Science 466 (2120), $2401-2423$

Gardiner, C.W., 2004. Handbook of Stochastic Methods: For Physics, Chemistry and the Natural Sciences. Springer Series in Synergetics. Springer.

Goyal, S., 2007. Connections: An Introduction to the Economics of Networks. Princeton University Press.

Hull, J., White, A., 2000. Valuing credit default swaps. I. No counterparty default risk. Journal of Derivatives 8 (1), 23.

Hull, J., White, A., 2001. Valuing credit default swaps. II. Modeling default correlations. Journal of Derivatives 8 (3), $12-22$.

Iori, G., De Masi, G., Precup, O., Gabbi, G., Caldarelli, G., 2008. A network analysis of the Italian overnight money market. Journal of Economic Dynamics and Control $32(1), 259-278$.

Iori, G., Jafarey, S., Padilla, F., 2006. Systemic risk on the interbank market. Journal of Economic Behaviour and Organisation 61 (4), $525-542$.

Jackson, M., 2008. Social and Economic Networks. Princeton University Press.

Jackson, M., Rogers, B., 2007. Relating network structure to diffusion properties through stochastic dominance. The BE Journal of Theoretical Economics 7

(1), 1-13.

Kiyotaki, N., Moore, J., 1997. Credit cycles. Journal of Political Economy 106, 211-248.

Kiyotaki, N., Moore, J., 2002. Balance-sheet contagion. The American Economic Review 92 (2), 46-50.

Kleinberg, J., 2007. Cascading behavior in networks: algorithmic and economic issues. Algorithmic Game Theory.

Kroszner, R.S., Laeven, L., Klingebiel, D., 2007. Banking crises, financial dependence, and growth. Journal of Financial Economics 84 (1), 187-228.

Longstaff, F., Schwartz, E., 1995. A simple approach to valuing risky fixed and floating rate debt. Journal of Finance 50 (3), $789-852$. 
Longstaff, F.A., 2010. The subprime credit crisis and contagion in financial markets. Journal of Financial Economics 97 (3), 436-450. (The 2007-8 financial crisis: lessons from corporate finance).

Merton, R., 1974. On the pricing of corporate debt: the risk structure of interest rates. The Journal of Finance 29 (2), $449-470$.

Mistrulli, P., 2011. Assessing financial contagion in the interbank market: maximum entropy versus observed interbank lending patterns. Journal of Banking \& Finance 35 (5), 1114-1127.

Morris, S., Shin, H., 2008. Financial regulation in a system context. In: Brookings Panel on Economic Activity, September.

Nier, E., Yang, J., Yorulmazer, T., Alentorn, A., 2007. Network models and financial stability. Journal of Economic Dynamics and Control 31 (6), 2033-2060.

Oksendal, B., Karsten, B., 1998. Stochastic Differential Equations: An Introduction with Applications. Springer.

Ongena, S., Smith, D., 2000. What determines the number of bank relationships? Cross-country evidence. Journal of Financial Intermediation 9 (1), 26-56.

Seneta, E., 2006. Non-Negative Matrices and Markov Chains. Springer.

Shin, H., 2008. Risk and liquidity in a system context. Journal of Financial Intermediation 17, 315-329.

Shleifer, A., Vishny, R.W., 2010. Unstable banking. Journal of Financial Economics 97 (3), 306-318. (The 2007-8 financial crisis: lessons from corporate finance).

Soramaki, K., Bech, M., Arnold, J., Glass, R., Beyeler, W., 2007. The topology of interbank payment flows. Physica A: Statistical Mechanics and its Applications 379 (1), 317-333.

Stiglitz, J. Contagion, liberalization, and the optimal structure of globalization. Journal of Globalization and Development, forthcoming.

Stiglitz, J., 2010. Risk and global economic architecture: why full financial integration may be undesirable. American Economic Review 100 (May (2)), 388-392.

Stiglitz, J.E., Greenwald, B.C.N., 2003. Towards a New Paradigm in Monetary Economics. Cambridge University Press, Cambridge.

Upper, C., Worms, A., 2004. Estimating bilateral exposures in the German interbank market: is there a danger of contagion? European Economic Review 48 (4), 827-849.

Vitali, S., Glattfelder, J., Battiston, S., 2011. The network of global corporate control. PLoS ONE 6 (10), e25995, http://dx.doi.org/10.1371/ journal.pone.0025995.

Wagner, W., 2010. Diversification at financial institutions and systemic crises. Journal of Financial Intermediation 19 (3), $373-386$. (Risk transfer mechanisms and financial stability).

Zhou, C., 1997. Default Correlation: An Analytical Result. Finance and Economics Discussion Series, May 27. Federal Reserve Board. 